\title{
Methylomic profiles reveal sex-specific differences in leukocyte composition associated with post-traumatic stress disorder
}

\author{
Grace S. Kim ${ }^{\mathrm{a}, \mathrm{b}}$, Alicia K. Smith ${ }^{\mathrm{c}, \mathrm{j}}$, Fei Xue ${ }^{\mathrm{d}}$, Vasiliki Michopoulos ${ }^{\mathrm{c}}$, Adriana Lori ${ }^{\mathrm{c}}$, \\ Don L. Armstrong ${ }^{\mathrm{e}}$, Allison E. Aiello ${ }^{\mathrm{f}}$, Karestan C. Koenen ${ }^{\mathrm{g}}$, Sandro Galea ${ }^{\mathrm{h}}$, Derek E. Wildman ${ }^{\mathrm{i}}$, \\ Monica Uddin ${ }^{i, *}$ \\ ${ }^{a}$ Neuroscience Program, University of Illinois at Urbana-Champaign, Urbana, IL, USA \\ ${ }^{\mathrm{b}}$ Medical Scholars Program, University of Illinois College of Medicine, Urbana, IL, USA \\ ${ }^{\mathrm{c}}$ Department of Psychiatry \& Behavioral Sciences, Emory University, Atlanta, GA, USA \\ ${ }^{\mathrm{d}}$ Department of Statistics, University of Illinois at Urbana-Champaign, Urbana, IL, USA \\ ${ }^{\mathrm{e}}$ Carl R. Woese Institute for Genomic Biology, University of Illinois at Urbana-Champaign, Urbana, IL, USA \\ ${ }^{\mathrm{f}}$ Gillings School of Global Public Health, University of North Carolina - Chapel Hill, Chapel Hill, NC, USA \\ ${ }^{\mathrm{g}}$ Department of Epidemiology, Harvard T.H. Chan School of Public Health, Boston, MA, USA \\ ${ }^{\mathrm{h}}$ Department of Epidemiology, Boston University School of Public Health, Boston, MA, USA \\ ${ }^{\mathrm{i}}$ Genomics Program, College of Public Health, University of South Florida, Tampa, FL, USA \\ ${ }^{\mathrm{j}}$ Department of Gynecology and Obstetrics, Emory University, Atlanta, GA, USA
}

A R T I C L E I N F O

\section{Keywords:}

PTSD

Monocytes

Leukocyte composition

DNA methylation

Sex differences

\begin{abstract}
A B S T R A C T
Post-traumatic stress disorder (PTSD) is a debilitating mental disorder precipitated by trauma exposure. However, only some persons exposed to trauma develop PTSD. There are sex differences in risk; twice as many women as men develop a lifetime diagnosis of PTSD. Methylomic profiles derived from peripheral blood are well-suited for investigating PTSD because DNA methylation (DNAm) encodes individual response to trauma and may play a key role in the immune dysregulation characteristic of PTSD pathophysiology. In the current study, we leveraged recent methodological advances to investigate sex-specific differences in DNAm-based leukocyte composition that are associated with lifetime PTSD. We estimated leukocyte composition on a combined methylation array dataset (483 participants, $\sim 450 \mathrm{k}$ CpG sites) consisting of two civilian cohorts, the Detroit Neighborhood Health Study and Grady Trauma Project. Sex-stratified Mann-Whitney U test and two-way ANCOVA revealed that lifetime PTSD was associated with significantly higher monocyte proportions in males, but not in females (Holm-adjusted p-val < 0.05). No difference in monocyte proportions was observed between current and remitted PTSD cases in males, suggesting that this sex-specific difference may reflect a long-standing trait of lifetime history of PTSD, rather than current state of PTSD. Associations with lifetime PTSD or PTSD status were not observed in any other leukocyte subtype and our finding in monocytes was confirmed using cell estimates based on a different deconvolution algorithm, suggesting that our sex-specific findings are robust across cell estimation approaches. Overall, our main finding of elevated monocyte proportions in males, but not in females with lifetime history of PTSD provides evidence for a sex-specific difference in peripheral blood leukocyte composition that is detectable in methylomic profiles and that may reflect long-standing changes associated with PTSD diagnosis.
\end{abstract}

\section{Introduction}

Post-traumatic stress disorder (PTSD) is a debilitating mental disorder that is precipitated by a traumatic experience involving direct or indirect exposure to actual or threatened death, serious injury, or sexual violence (American Psychiatric Association, 2013). PTSD presents with intrusive and persistent re-experiencing of the traumatic event, avoidance of distressing, trauma-associated stimuli, negative alterations in cognition and mood, and alterations in arousal/reactivity that persist for longer than a month (American Psychiatric Association, 1994; 2013). While most individuals are exposed to a potentially traumatic event at some point in their lives, only some develop PTSD (Breslau,

\footnotetext{
* Corresponding author at: Genomics Program, College of Public Health, University of South Florida, 3720 Spectrum Blvd., Ste. 304, Tampa, FL 33612, USA.

E-mail address: monica43@health.usf.edu (M. Uddin).
} 
2009; Kessler et al., 1995; Kilpatrick, 2013; Liu, 2017; Benjet, 2016; Kessler, 2005; Kessler and Wang, 2008), suggesting that the disorder reflects a distinct inability to reinstate homeostasis after psychological trauma in vulnerable individuals (Yehuda et al., 2011).

Epidemiological studies have identified sex to be a significant vulnerability factor for developing PTSD, with women twice as likely to have lifetime PTSD than men, even when risk of exposure and types of trauma are taken into consideration (Kessler et al., 1995; Kilpatrick, 2013; Kessler and Wang, 2008; Breslau, 1998; Breslau et al., 1997; Tolin and Foa, 2006). This sex bias in disease prevalence is also observed in other stress-related mood and anxiety disorders (Kessler, 1994), including depression (Kessler et al., 1993). Preclinical and clinical investigations have identified sexual dimorphism in stress response systems that may be involved in the increased prevalence of stress-related psychopathologies in women (Hodes, 2013; Bangasser and Valentino, 2014). Furthermore, in addition to sexual dimorphism in the neurobiological underpinnings of stress/trauma response, recent animal studies suggest that behavioral response to traumatic stress is fundamentally different $\mathrm{b}$ etween $\mathrm{m}$ ales a nd $\mathrm{f}$ emales a nd $\mathrm{s}$ hould be considered in interpretation of results (Pooley, 2018). In humans, response to stress/trauma exposure involves both biological and social contributors corresponding to sex and gender-related variables. While the effects of sex and gender are difficult to disentangle, investigations stratified by biological sex, understood to interact with gender-related variables, are warranted to improve our currently limited understanding of the sex-specific biological processes dysregulated in PTSD pathophysiology.

Mounting evidence suggests a key role for stress-induced inflammation a nd i mmune a lteration in $\mathrm{t}$ he $\mathrm{d}$ evelopment a nd maintenance of PTSD and other stress-related psychiatric disorders. Although findings from human literature have been mixed, PTSD has generally been associated with increased pro-inflammatory tone, basally and in response to immune challenge, via both cytokine signaling and changes in immune cell distribution/function (Passos, 2015; Michopoulos et al., 2017; Wang and Young, 2016; Kawamura et al., 2001; Altemus et al., 2003; G lover et al., 2005; G ill et al., 2009; G otovac, 2010; Lindqvist, 2014; Aiello, 2016; Lindqvist, 2017; Sondergaard et al., 2004; Sommershof, 2009; Hoge, 2009; Michopoulos et al., 2015; Bersani, 2016; Wang et al., 2016). Investigations in animal models, primarily in male rodent studies, have provided mechanistic insights into how peripheral immune cell response/signaling and distribution is linked with microglial activation and neuroinflammatory dynamics to trigger stress-induced anxiety behavior and memory impairment (Wohleb, 2011; Wohleb et al., 2013; Wohleb, 2014; McKim, 2016a,b; Wohleb and Delpech, 2017; Yin, 2019).

Epigenetic mechanisms have emerged as important regulators of PTSD-associated immune dysregulation and inflammation (Bam, 2016; Zhou, 2014; Uddin, 2010; Rusiecki, 2013; Bam, 2016; Smith, 2011), and are particularly significant f or the study of P TSD b ecause they capture the interactions among pre-disposing genetic/environmental risk factors and the precipitating trauma exposure. Individual response to trauma exposure can be encoded as short-lived or persistent epigenetic changes that reflect and $m$ ay contribute to posttraumatic physiological changes, some of which remain after remission of PTSD symptoms. DNA methylation (DNAm) has been the most widely studied epigenetic mechanism and evidence from both animal and human models point to its key role in stress regulation (Yehuda et al., 2011; Weaver, 2004; McGowan, 2009; Stankiewicz et al., 2013), fear memory (Miller et al., 2008; Maddox et al., 2013; Zovkic and Sweatt, 2013; Malan-Muller et al., 2014; Kwapis and Wood, 2014), and immune function (Uddin, 2010; Rusiecki, 2013; Bam, 2016; Smith, 2011; Alvarez-Errico et al., 2015; Sun, 2016; Chen, et al., 2016; Wolf, 2018), in both brain and blood. Exploring PTSD-associated DNAm profiles in blood may inform our understanding of mechanisms underlying immune dysregulation, particularly those that coordinate peripheral immune-neuroimmune crosstalk (Irwin and Cole, 2011; Pfau and Russo,
2015); moreover, given the well-known sex difference in PTSD prevalence and in immune response (Klein and Flanagan, 2016; Osborne et al., 2018), sex-stratified investigation relating to these dual factors is warranted to identify potential differences in variability by sex that may be missed in analyses combining both sexes.

Peripheral blood-based methylomic profiles are comprised of two dynamic components: 1) profiles reflecting proportions of immune cell subtypes (i.e., leukocyte composition) and 2) alterations in DNAm levels at $\mathrm{CpG}$ sites genome-wide (i.e., differential methylation). Epigenome-wide association studies (EWAS) often seek to identify dynamic differential methylation marks and treat cellular heterogeneity as a major confound that must be addressed to improve signal detection. However, differences in leukocyte subtypes provide key insights into immunological changes and warrant examination themselves. Recent developments in deconvolution algorithms and cell-type discriminating reference databases have improved estimates (Titus et al., 2017; Teschendorff et al., 2017; Koestler, 2016; Salas, 2018) and enabled utility of DNAm-based leukocyte subtype estimates as proxies for white blood cell differential-based metrics (Wiencke, 2017; Koestler, 2017). Leveraging these recently developed methods, here we use leukocytederived methylomic profiles combined from two civilian cohorts to investigate our hypothesis that PTSD is associated with sex-specific differences in leukocyte composition, detectable by DNAm-based estimates. To our knowledge, this study is the first to investigate leukocyte composition profiles in PTSD using these new DNAm-based approaches for immune profiling.

\section{Materials and methods}

\subsection{Study participants}

Samples from trauma-exposed, adult participants with available Illumina HumanMethylation450 (450 K) BeadChip array data were selected from two predominantly African-American, community-based cohorts examining biological and environmental correlates of PTSD, namely the Detroit Neighborhood Health Study (DNHS; $\mathrm{n}=192$ ) and Grady Trauma Project (GTP; $n=422$ ). The DNHS, based in Detroit, MI, was approved by the institutional review boards of the University of Michigan and University of North Carolina at Chapel Hill. The GTP, based in Atlanta, GA, was approved by the institutional review boards of Emory University School of Medicine and Grady Memorial Hospital. All participants provided written informed consent prior to data collection. Details regarding the DNHS (Uddin, 2010; Goldmann, 2011; Meyers, 2015) and GTP (Gillespie, 2009; Binder, 2008; Zannas, 2015) were published previously. While neither study excluded participants based on illness, women known to be pregnant (in the GTP) were excluded from estimation and analyses, due to well-known/known significant differences in leukocyte composition during pregnancy (Luppi, 2003). Collected demographic data included self-reported gender, race, age, and current smoking, which was defined as any cigarette smoking in the past 30 days.

\subsection{Assessment of PTSD}

Study participants were assessed for PTSD, as defined by the Diagnostic and Statistical Manual of Mental Disorders, Fourth Edition (DSM-IV) (American Psychiatric Association, 2013). In the DNHS, study participants were assessed for PTSD using the well-validated self-report PTSD Checklist, Civilian Version (PCL-C) (Blanchard et al., 1996; Grubaugh et al., 2007; Wilkins et al., 2011; Ruggiero et al., 2003) and additional questions about duration, timing, and impairment due to symptoms, via structured telephone interviews (Breslau, 1998; Uddin, 2010; Koenen, 2011). Participants who met all six DSM-IV criteria in reference to their worst traumatic event or to a randomly selected traumatic event (if the participant experienced more than one trauma), were considered affected by lifetime PTSD. Those that met criteria 
based on symptoms reported within the past month were considered affected by current PTSD. Analysis of data from the clinical interviews showed that the PTSD instrument used during structured telephone interviews had excellent internal consistency and high concordance (Uddin, 2010; Uddin, 2011). The PCL-C yielded a Cronbach coefficient alpha ( $\alpha$ ) of 0.93 . Using cluster scoring based on DSM-IV criteria (i.e. to be a case, the participant's symptoms had to meet all six criteria), the instrument had a specificity (SP) of 0.97 , sensitivity (SE) of 0.24 , positive predictive value (PPV) of 0.80 , negative predictive value (NPV) 0.72 , and an area under the ROC curve (AUC) of 0.76 , as previously reported (Uddin, 2010; Uddin, 2011). In the GTP (Jovanovic, 2010), study participants were assessed for lifetime and current PTSD using the Clinician-Administered Post-traumatic Stress Disorder Scale (CAPS, DSM-IV), a structured interview administered by a clinician that has previously been shown to have excellent reliability (i.e., consistent scores across items, raters, testing occasions) and excellent convergent and discriminant validity in large scale psychometric studies (Blake, 1995; Weathers et al., 2001). For lifetime analyses, participants meeting criteria for PTSD at any point (including current and past PTSD) were considered cases and compared to trauma-exposed controls who lacked a history of PTSD at any point in their lifetime. For comparisons of current PTSD with remitted PTSD and trauma exposed controls, participants with lifetime PTSD were further separated into two groups: those with current PTSD and those with lifetime (but not current) PTSD.

\subsection{Sample processing}

In the DNHS, samples were obtained via an in-home blood draw performed by a clinician, processed in the lab within two hours, and stored at $-20^{\circ} \mathrm{C}$ until testing. Detailed methods regarding biospecimen processing in the DNHS are available in a separate publication (Weckle, 2015). In the GTP, DNA was extracted from whole blood, aliquoted, and frozen at $-80^{\circ} \mathrm{C}$ within two hours of collection. G enomic DNA was extracted from peripheral blood using the DNA Mini Kit (Qiagen, Germantown, MD) in the DNHS and the Gentra Puregene Kit (Qiagen, Germantown, MD) in the GTP. Both studies bisulfite-converted the DNA samples using the Zymo EZ-96 DNA methylation kit (Zymo Research, Irvine, CA) and used $500 \mathrm{ng}$ of DNA per sample for whole-genome amplification, fragmentation, and hybridization to the Illumina Human Methylation 450 K BeadChip array (Illumina, San Diego, CA), according to the manufacturers' recommended protocols. Sample processing procedures have been published previously for both the DNHS and GTP (Wolf, 2018; Zannas, 2015; Ratanatharathorn, 2017; Uddin, 2018; Mehta, 2013).

\subsection{Quality control and pre-processing of $450 \mathrm{~K}$ data}

The raw .idat files $\mathrm{w}$ ere $\mathrm{i}$ mported i nto $\mathrm{R}$ ( version 3 .5.1) ( $\mathrm{R}$ Foundation for Statistical Computing, Vienna, Austria, 2018), using the minfi package (Aryee, 2014), a vailable on Bioconductor (version 3.7) (Huber, 2015; Gentleman, 2004), for all subsequent data processing and analyses. After quality control (QC) (Aryee, 2014; Barfield et al., 2012; Xu et al., 2016), data pre-processing (Liu and Siegmund, 2016; Fortin et al., 2017; Chen, 2013; Teschendorff, 2013; Morris, 2014; Tian, 2017) was conducted on all QC'ed samples (DNHS: $n=187$; GTP: $\mathrm{n}=416)$. This included duplicates $(\mathrm{n}=12)$ in the DNHS and participants with known pregnancy $(n=26)$ or missing PTSD phenotype data $(n=82)$ in the GTP. Analyses were conducted on unique participants that passed QC, as described below, and had PTSD data available (Table 1).

For data quality assessment, samples were checked for 1) low total signal (mean signal intensity less than half of the overall median, after setting probes with detection p-value $>0.001$ or $<2,000$ arbitrary units to missing); 2) $>1 \%$ of failed probes (detection p-value $>$ 0.001 ); 3) outlying beta value distribution (i.e., smaller than three
Table 1

Key demographic characteristics, by Sex.

\begin{tabular}{|c|c|c|c|}
\hline & Female $(n=330)$ & $\operatorname{Male}(\mathbf{n}=153)$ & $\operatorname{Total}(n=483)$ \\
\hline \multicolumn{4}{|l|}{ Study } \\
\hline DNHS & $111(33.6 \%)$ & $64(41.8 \%)$ & $175(36.2 \%)$ \\
\hline GTP & $219(66.4 \%)$ & $89(58.2 \%)$ & $308(63.8 \%)$ \\
\hline \multicolumn{4}{|l|}{ Race } \\
\hline AA & $291(88.2 \%)$ & $140(91.5 \%)$ & $431(89.2 \%)$ \\
\hline $\mathrm{CA}$ & $33(10.0 \%)$ & $11(7.2 \%)$ & $44(9.1 \%)$ \\
\hline Other & $6(1.8 \%)$ & $2(1.3 \%)$ & $8(1.7 \%)$ \\
\hline \multicolumn{4}{|c|}{ Median Age } \\
\hline & $49.0(38.0-55.0)$ & $48.0(36.0-56.0)$ & $48.0(37.5-55.0)$ \\
\hline \multicolumn{4}{|c|}{ Current Smoking } \\
\hline no & $210(63.6 \%)$ & $72(47.1 \%)$ & $282(58.4 \%)$ \\
\hline yes & $109(33.0 \%)$ & $78(51.0 \%)$ & $187(38.7 \%)$ \\
\hline Missing & $11(3.3 \%)$ & $3(2.0 \%)$ & $14(2.9 \%)$ \\
\hline \multicolumn{4}{|c|}{ Lifetime PTSD } \\
\hline no & 135 (40.9\%) & $71(46.4 \%)$ & $206(42.7 \%)$ \\
\hline yes & $195(59.1 \%)$ & $82(53.6 \%)$ & $277(57.3 \%)$ \\
\hline
\end{tabular}

This table describes the subset of participants included in primary analyses investigating sex-specific associations between DNAm-based cell estimates and lifetime PTSD, by sex. For the 2-way ANCOVA, 14 participants were excluded due to missing current smoking data.

DNHS: Detroit Neighborhood Health Study; GTP: Grady Trauma Project

times interquartile range (IQR) from the lower quartile or $>3$ times IQR from the upper quartile); 4) greater than three standard deviations of the mean bisulfite conversion control probe signal intensity (Salas, 2018). Additionally, samples were checked for gender discordance based on median total intensity of $\mathrm{X}$ and $\mathrm{Y}$-chromosome mapped probes (as implemented in minfi) (Aryee, 2014) and removed if predicted sex differed from self-reported gender. Five samples were removed among DNHS samples for gender discordance, and six samples were removed among GTP samples (two for data quality and four for gender discordance). After within-array background correction and dye-bias equalization using out-of-band control probes (ssNoob, minfi) (Fortin et al., 2017; Triche et al., 2013), probes with detection p-value > 0.001 in $>10 \%$ of samples (Barfield et al., 2012) and cross-reactive probes (Chen, 2013) (i.e., cross-hybridized between autosomes and sex chromosomes) were removed. Beta-mixture quantile (BMIQ) normalization (ChAMP) (Morris, 2014; Tian, 2017) was used to correct for type II probe bias (Teschendorff, 2013).

To control for technical artifacts (e.g., sample processing and imaging batch effects), principal components (PCs) based on non-negative control probe signal intensity (Xu et al., 2016) were removed from BMIQ-normalized M-values (i.e., logit-transformed beta-values) separately for each study. PC correlation heatmaps were used to check for successful removal/reduction of batch effects, especially chip and row effects, while maintaining signal from biological variables. The DNHS and GTP datasets were then combined and an empirical Bayes method (i.e., ComBat (Johnson et al., 2007) in the sva package (Leek et al., 2012) was used on the combined M-values to account for study effects while controlling for sex and age. Data quality assessment, QC probe filtering, and first step of batch removal were study-specific, while preprocessing steps (ssNoob + BMIQ) implemented within-array approaches unaffected by study. Only probes that passed QC in both studies ( $n=455,072$ probes) were included in the combined dataset.

\subsection{Leukocyte composition estimation}

Leukocyte composition was estimated on ComBat-adjusted betavalues using the EpiDISH (Teschendorff et al., 2017) reference database, which is informed by cell-type specific DNase hypersensitive sites (DHS; based on the NIH Epigenomics Roadmap database (Roadmap Epigenomics, 2015)) and is optimized for discriminating granulocytes, 

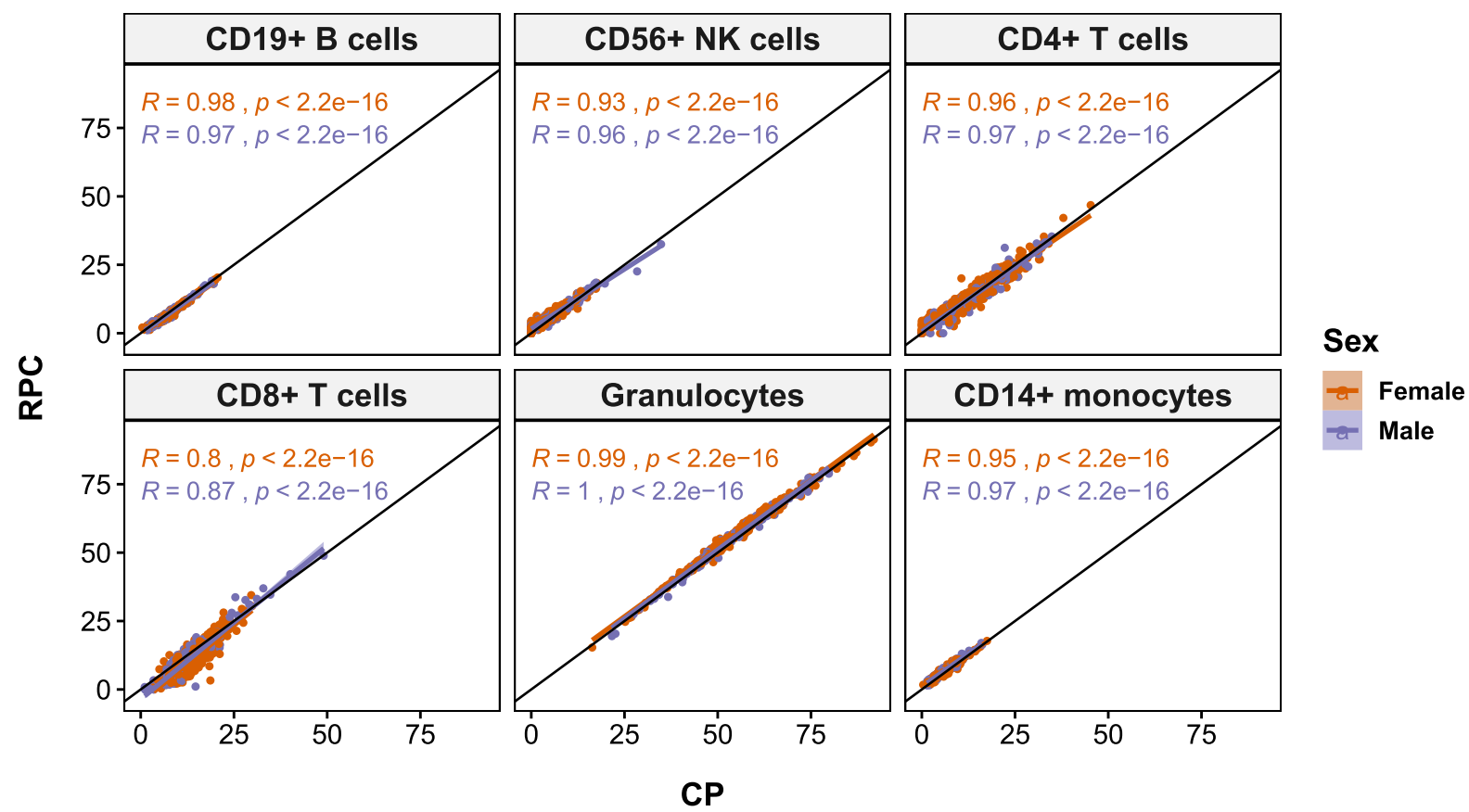

Fig. 1. Correlation (Spearman's rho) between cell estimates derived using robust partial correlation (RPC) and constrained projection (CP) deconvolution algorithms is high in all leukocyte subtypes, with CD8 ${ }^{+} \mathrm{T}$ cells (CD8T) showing the worst agreement at $R=0.8$ in females and $R=0.87$ in males. CD8T cells also showed the largest discrepancy in sex-specific correlation of estimates. RPC-CP correlation was $>0.9$ and difference in RPC-CP correlation between sexes was between 0.01 and 0.03 for all other leukocyte subtypes.

$\mathrm{CD}^{+} 4^{+}$monocytes, $\mathrm{CD}^{+}{ }^{+} \mathrm{T}$ cells, $\mathrm{CD}^{+}{ }^{+} \mathrm{T}$ cells, $\mathrm{CD} 19^{+} \mathrm{B}$ cells, and $\mathrm{CD}^{+} 6^{+}$natural killer cells. Robust partial correlation (RPC; robust multivariate linear regression, non-constrained projection) was used as the primary deconvolution algorithm and EpiDISH's implementation of linear, constrained projection (CP), originally introduced by Houseman (2012), was used to calculate a second set of estimates for comparison.

\subsection{Ancestry estimation}

DNAm-based ancestry PCs were derived on cleaned beta-values after regressing out sex and age from batch-adjusted M-values. Ancestry PCs were calculated on a subset of 2,317 ancestry informative CpG probes included in two published ancestry informative $\mathrm{CpG}$ lists that accounted for confounders (Rahmani, 2017) and that included probes within 10 base pairs (bp) of single nucleotide polymorphisms (SNPs) (Barfield, 2014). The first 2 PCs based on this subset of probes were used as ancestry PCs (ancPCs) after checking for strong association with self-reported race and effective separation of self-reported races.

\subsection{Statistical analysis}

The Shapiro-Wilk test was used to assess normality and Levene's test was used to compare equality of variance among groups. Since cell estimates had dissimilar, non-normal distributions, non-parametric tests were used for all initial group comparisons of leukocyte subtypes. The two-sample Kolmogorov-Smirnov (KS) test was used to compare distribution of cell estimates when variances were unequal between groups and Mann-Whitney U test was used to compare mean ranks of cell estimates otherwise. Spearman's rank correlation was used to assess agreement between estimates based on RPC and CP deconvolution approaches. A threshold of 0.05 was used for p-values and p-values were adjusted for multiple comparisons using Holm's method (Holm, 1979), unless otherwise specified.

To test our main hypothesis-that PTSD is associated with sexspecific differences in leukocyte composition-initial sex-stratified analyses were conducted on all leukocyte subtypes using the non- parametric Mann-Whitney U test. For leukocyte subtypes determined to be significantly associated with lifetime PTSD in either sex based on initial Mann-Whitney U tests, a two-way analysis of covariance (ANCOVA; Type III) controlling for age, ancestry (based on DNAm ancestry PCs), and current smoking, was performed with post-hoc comparison of estimated marginal means to examine the effects of sex and lifetime PTSD on transformed cell estimates. Transformation for cell estimate was conducted to meet modeling assumptions for ANCOVA and was informed by Tukey's Ladder of Powers. Power parameter $(\lambda)$ was computed to maximize normality based on the Shapiro-Wilks W statistic. Sex-stratified Kruskal-Wallis and post-hoc Dunn tests were conducted as additional follow-up to investigate possible differences in cell proportions by PTSD status (i.e., trauma-exposed controls, remitted PTSD, and current PTSD).

\section{Results}

\subsection{Demographic characteristics of sampled study participants from the DNHS and GTP}

The demographic characteristics of study participants included in primary analyses investigating sex-specific associations between DNAm-based cell estimates and lifetime PTSD are presented in Table 1. Of the 483 participants from the combined DNHS and GTP sample, $57.3 \%$ had a lifetime diagnosis of PTSD, $68.3 \%$ were female, and $38.7 \%$ were current smokers. The study population was predominantly African-American (89.2\%), based on self-reported race, and the median age was 48 years (IQR: 17.5 ; $37.5-55$ years).

\subsection{Comparison of leukocyte subtype estimates by deconvolution approach}

Good overall agreement was observed between RPC and CP estimates, as measured by Spearman's correlation (i.e., RPC-CP correlation), but $\mathrm{CD}^{+} \mathrm{T}$ cells (CD8T) showed much poorer agreement, $\rho_{s}(481)=0.83$, relative to the other leukocyte subtypes, $\rho_{s}(481)>0.94$ (Fig. 1). Since the main objective of this study was to 
investigate sex-specific differences in leukocyte composition, comparison of RPC and CP estimates was stratified by sex. Sex-stratified RPC$\mathrm{CP}$ correlation revealed that the largest difference in RPC-CP correlation between sexes was also found in CD8T cells, $\left|\Delta \rho_{s}\right|=0.07$, such that females showed poorer correlation, $\rho_{s}(328)=0.80$, than males, $\rho_{s}(151)=0.87$. For the other leukocyte subtypes, the difference in RPC-CP correlation between sexes $\left(\left|\Delta \rho_{s}\right|\right)$, ranged from 0.01 to 0.03 , with $\mathrm{CD}_{56}{ }^{+}$natural killer (NK) cells having the second largest difference in correlation between sexes (female: $\rho_{s}(328)=0.93$; male: $\left.\rho_{s}(151)=0.96\right)$. In all leukocyte subtypes, except CD19 ${ }^{+}$B cells, females had lower correlation coefficients than males. Detailed results for RPC-based cell estimates are reported below and corresponding results based on CP-based estimates are reported in supplementary materials, due to strong agreement between findings from both sets of estimates.

\subsection{Comparison of leukocyte subtype estimates by sex and lifetime PTSD}

Cell estimates were compared by sex, lifetime PTSD, and study in each leukocyte subtype to establish overall differences. Significant overall sex differences were observed in the distributions of NK (KS: $D=0.19$, adj. $p=0.007$ ) and CD8T cell proportions (KS: $D=0.16$; adj. $p=0.04$ ) in RPC estimates. Males showed greater variability than females for both NK and CD8T cells (male vs. female - IQR $\mathrm{NK}_{\mathrm{NK}}: 6.2 \%$ vs. $4.15 \%$; $\mathrm{IQR}_{\mathrm{CD} 8 \mathrm{~T}}$ : $9.5 \%$ vs. $\left.6.2 \%\right)$, as well as higher median NK (5.5\% vs. $4.4 \%)$ and lower median CD8T (9.0\% vs. 9.7\%) cell proportions (Fig. 2). No significant overall differences (i.e., in both sexes combined) were observed between lifetime PTSD cases and trauma-exposed controls in any leukocyte subtype (Mann-Whitney). Additional analyses comparing leukocyte subtype proportions between participating cohorts and assessing age effects in each cell type are reported in supplementary materials.

\subsection{Elevated monocyte proportions were associated with lifetime PTSD in} males, but not females

Sex-stratified Mann-Whitney U tests r evealed a significant difference in monocyte proportions between PTSD cases and controls in males (Figs. 3 and 4). Males with lifetime PTSD had higher median monocyte proportions than trauma-exposed controls, $U=2100$, $Z=-2.9, p=0.004$, adj. $p=0.026, r=0.23$. In contrast, no difference in monocyte estimates was found between groups in females, $U=13000, Z=-0.58, p=0.6$, adj. $p=1, r=0.03$. Lifetime PTSDassociated differences were not observed in any other leukocyte subtypes in either sex.

A two-way ANCOVA was conducted to investigate whether sex moderated the effects of lifetime PTSD on transformed monocyte estimates, while accounting for age, ancestry, and current smoking (Table 2). Monocyte estimates were square root transformed for the ANCOVA to meet model assumptions (i.e., normality) and were informed by Tukey's Ladder of Powers (RPC: $\lambda=0.43$; CP: $\lambda=0.5$ ). A significant interaction was found between sex and lifetime PTSD, $F(1$, $461)=4.89, p=0.027, \eta_{\mathrm{p}}^{2}=0.011$. Post-hoc comparison of estimated marginal means (EMMs) for lifetime PTSD by sex (Fig. 5; Table 3) showed a significant mean difference between lifetime PTSD cases and controls in males, $\Delta E M M=0.26, S E=0.08, t(461)=3.32, p=0.001$, where mean monocyte estimates were higher in lifetime PTSD cases than controls. No significant mean difference was observed between PTSD cases and controls in females, $\triangle E M M=0.05, S E=0.05, t$ $(461)=0.89, p=0.37$, confirming findings from initial sex-stratified analyses. Together, our results suggest that male PTSD cases have significantly elevated monocyte proportions compared to trauma-exposed controls and that this lifetime PTSD-associated difference is not observed in females.

3.5. Association between monocyte proportions and lifetime PTSD in males is independent of current PTSD status

To investigate whether participants with current PTSD exhibited a different monocyte profile from those with remitted PTSD, a sex-stratified Kruskal-Wallis test was conducted for PTSD status (i.e., traumaexposed controls, remitted PTSD, and current PTSD; Fig. 6). A significant difference in monocyte estimates was observed in males, $H$ (2) $=8.2, p=0.017$, but not in females, $H(2)=1.1, p=0.59$, confirming findings from analyses for lifetime PTSD. The post-hoc Dunn test revealed significant differences between PTSD case groups and trauma-exposed controls (current PTSD vs. controls: $Z=2.31$,
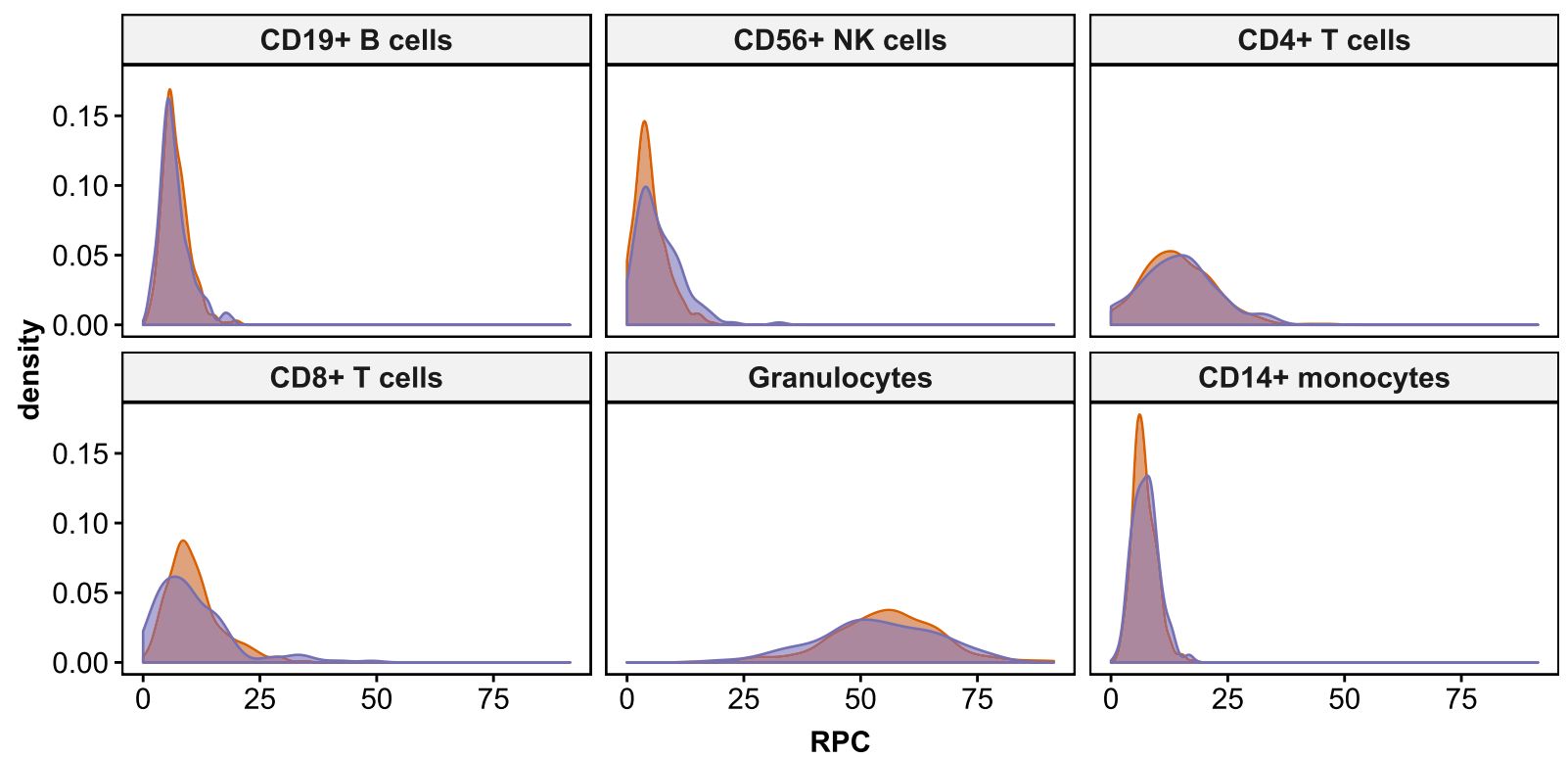

Sex $\square$ Female $\square$ Male

Fig. 2. Distribution of leukocyte subtypes based on robust partial correlation (RPC) estimates, by sex. Sex differences in $\mathrm{CD}^{+}{ }^{+}$and $\mathrm{CD}^{+} 6^{+} \mathrm{NK}$ cell distributions were found to be prominent. 

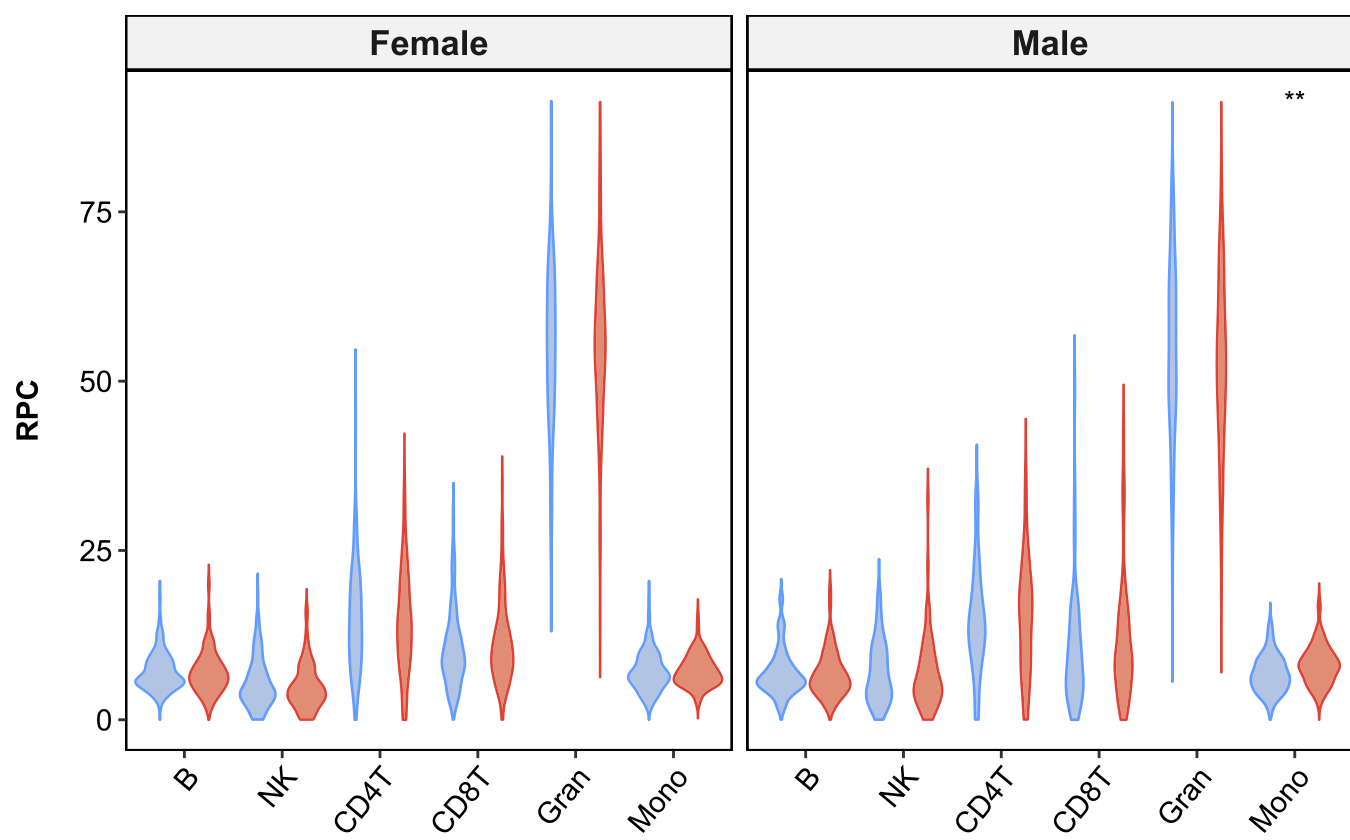

\section{PTSD Status}

no

Fig. 3. Violin plots of RPC estimates for lifetime PTSD cases and controls, stratified by sex. Only monocyte proportions in males showed a statistically significant difference between lifetime PTSD cases and controls, based on Mann-Whitney $U$ test (p-value $=0.004$; Holm-adjusted p-value $=0.026$ ). For figure labels on x-axis: $\mathrm{B}=\mathrm{CD} 19^{+} \mathrm{B}$ cells; $\mathrm{NK}=\mathrm{CD}_{6}{ }^{+} \mathrm{NK}$ cells; $\mathrm{CD} 4 \mathrm{~T}=\mathrm{CD} 4^{+} \mathrm{T}$ cells; $\mathrm{CD} 8 \mathrm{~T}=\mathrm{CD} 8^{+} \mathrm{T}$ cells; Gran $=$ Granulocytes; Mono $=\mathrm{CD} 14^{+}$monocytes.

$p=0.021$, adj. $p=0.042, r=0.23$; remitted PTSD vs. controls: $Z=2.40, p=0.016$, adj. $p=0.049, r=0.22$ ), but no significant difference between current and remitted PTSD groups, $Z=0.18, p=0.86$, adj. $p=0.86, r=0.02$. These findings suggest that the association between monocyte proportions and lifetime PTSD in males is independent of current PTSD state and may reflect long-standing changes associated with lifetime history of PTSD diagnosis.

Comparative analyses based on CP monocyte estimates showed similar results to RPC-based results (see Supplementary Materials); however, CP-based results consistently presented smaller effect sizes than RPC-based results across all analyses and follow-up comparisons between PTSD case groups and trauma-exposed controls did not reach significance after p-value adjustment in post-hoc Dunn test using CP monocyte estimates in males.
Table 2

Two-way ANCOVA Table for RPC monocyte estimates $(n=469)$.

\begin{tabular}{lllllll}
\hline Terms & $\begin{array}{l}\text { Type III Sum of } \\
\text { Squares }\end{array}$ & $d f$ & $\begin{array}{l}\text { Mean } \\
\text { Square }\end{array}$ & $F$ & $p$ & partial $\eta^{2}$ \\
\hline Sex & 0.336 & 1 & 0.336 & 1.469 & 0.226 & 0.003 \\
PTSDlife & 2.379 & 1 & 2.379 & 10.407 & $\begin{array}{l}0.001^{*-} \\
* *\end{array}$ & 0.022 \\
& & & & & & \\
Age & 2.309 & 1 & 2.309 & 10.101 & $\begin{array}{l}0.002^{*}- \\
* *\end{array}$ & 0.021 \\
& & & & & & \\
ancPC1 & 0.000 & 1 & 0.000 & 0.001 & 0.977 & 0.000 \\
ancPC2 & 0.219 & 1 & 0.219 & 0.957 & 0.329 & 0.002 \\
Smoking & 0.060 & 1 & 0.060 & 0.261 & 0.610 & 0.001 \\
Sex:PTSDlife & 1.146 & 1 & 1.146 & 5.011 & $0.026^{*}$ & 0.011 \\
Residuals & 105.399 & 461 & 0.229 & & & \\
\hline
\end{tabular}

$* * p<0.05, * * p<0.01, * * * p<0.005$.

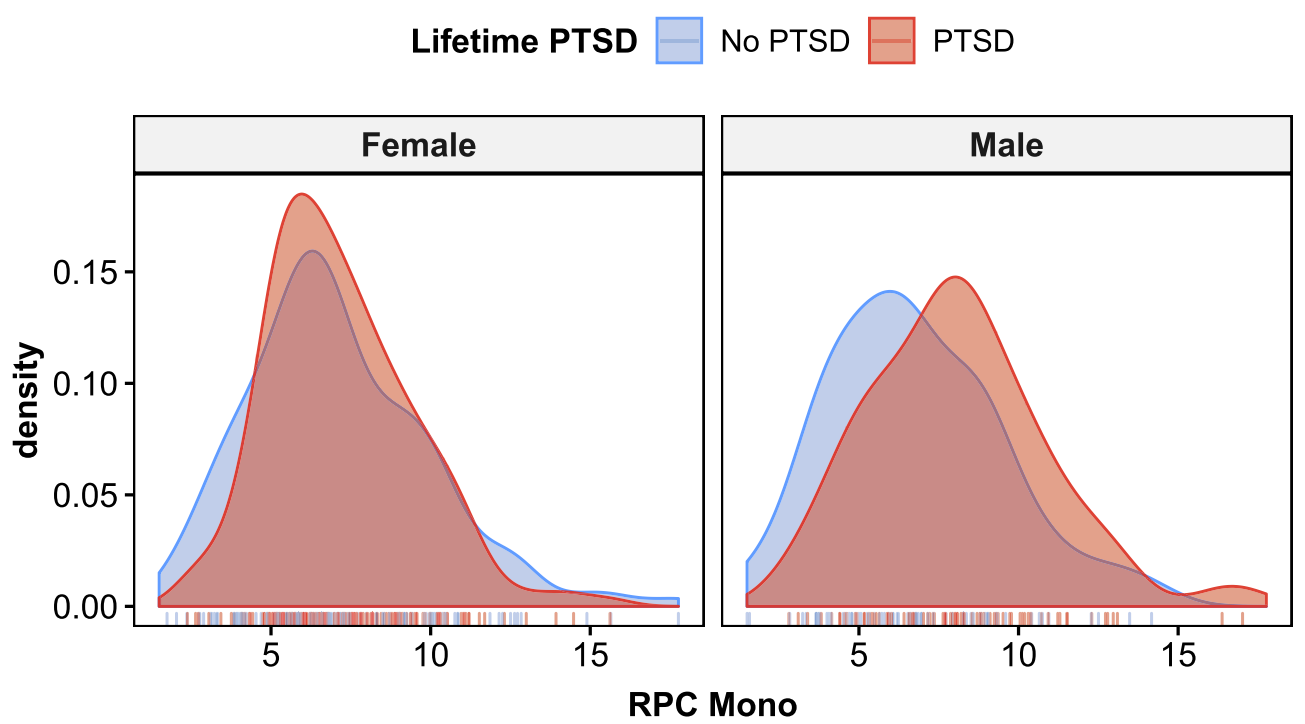

Fig. 4. Density plots for RPC monocyte estimates in lifetime PTSD cases and controls, stratified by sex, show distinctly higher monocyte levels in males with lifetime PTSD compared to trauma-exposed controls. This difference in monocyte levels between lifetime PTSD cases and controls is not observed in females. 


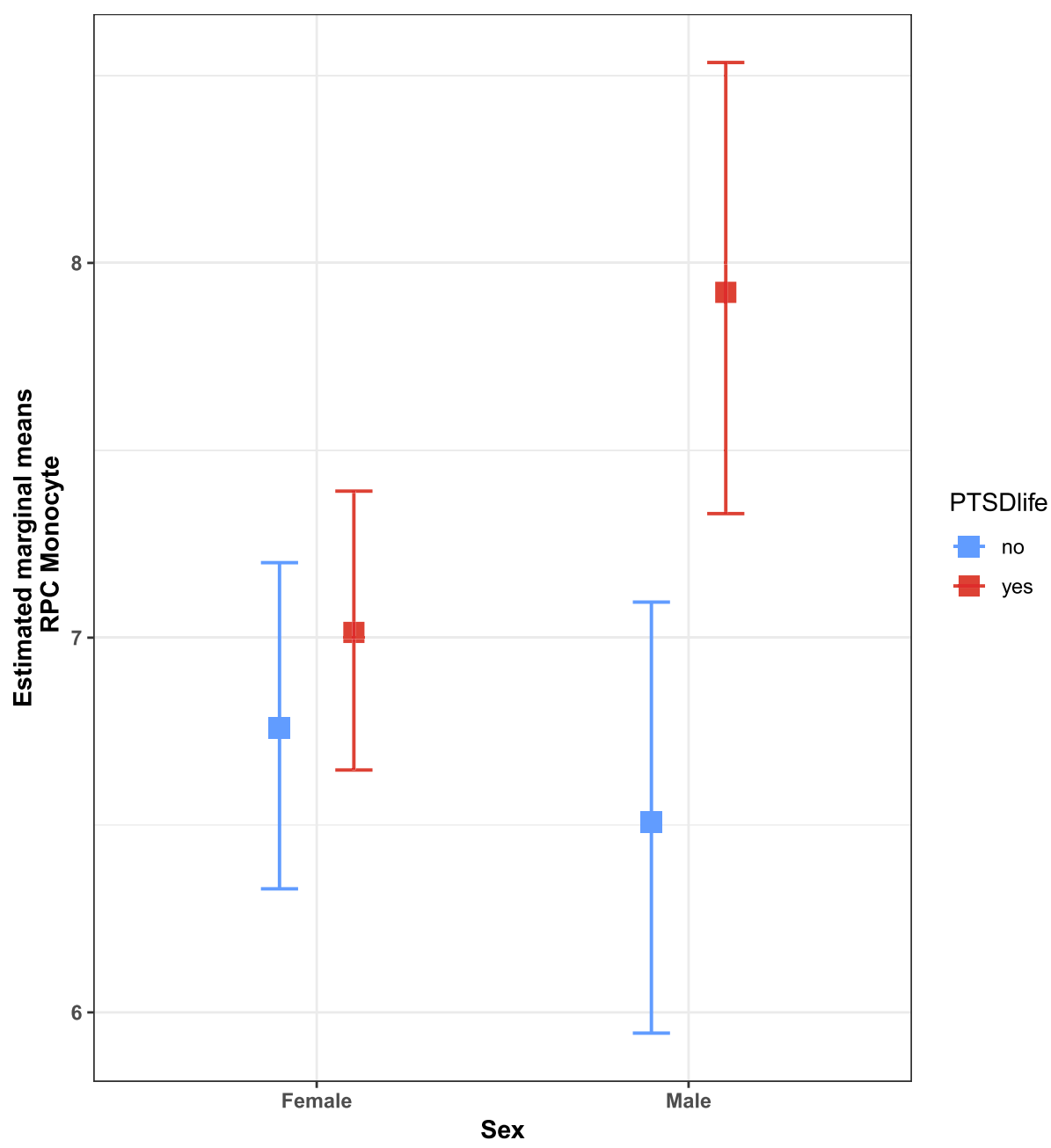

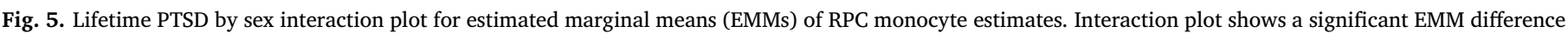

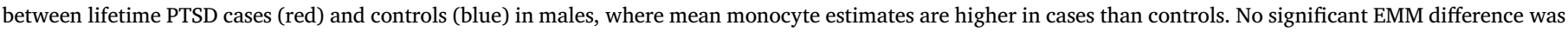

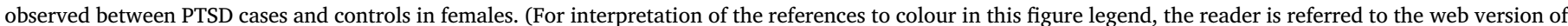
this article.)

Table 3

Summary for RPC monocyte estimates by group.

\begin{tabular}{lllllllll}
\hline Sex & PTSD & $\mathrm{n}$ & mean & $\mathrm{SE}$ & $\mathrm{EMM}$ & $\mathrm{SE}_{\mathrm{EMM}}$ & lower.CL & upper.CL \\
\hline Female & no & 135 & 7.113 & 0.2443 & 6.758 & 0.2214 & 6.330 & 7.200 \\
Female & yes & 184 & 7.182 & 0.1691 & 7.014 & 0.1893 & 6.647 & 7.391 \\
Male & no & 70 & 6.803 & 0.3228 & 6.507 & 0.2926 & 5.945 & 7.095 \\
Male & yes & 80 & 8.103 & 0.3147 & 7.921 & 0.3063 & 7.331 & 8.535 \\
\hline
\end{tabular}

This table describes untransformed RPC monocyte estimates by group (i.e., sex and lifetime PTSD); $\mathrm{n}=$ count per group; EMM = estimated marginal means (i.e., least squares means); $\mathrm{SE}=$ standard errors for regular means; $\mathrm{SE}_{\mathrm{EMM}}=$ standard errors for EMM.

Lower and upper confidence limits (CL) are for 95\% level. EMM and intervals were back-transformed from the square root scale to the original scale of cell subtype proportions (\%). Significance level of alpha $=0.05$ was used for EMM comparisons. Results for pairwise comparison were averaged over levels for current smoking. Degree of freedom was 461 and male lifetime PTSD cases were significantly different from other groups.

\section{Discussion}

Methylomic profiles derived from peripheral blood offer a wealth of information and can be harnessed to detect two dynamic measures of immune state: 1) differences in leukocyte composition (i.e., proportions of peripheral immune cell subtypes); and 2) true alterations in methylation involved in epigenetic regulation of immune processes. They are particularly well-suited for investigating PTSD because DNAm encodes individual response to trauma and may play a key role in PTSD-associated immune dysregulation. Given the prominent sex differences in both PTSD prevalence (Kessler et al., 1995; Kilpatrick, 2013; Kessler and Wang, 2008; Breslau, 1998; Breslau et al., 1997; Tolin and Foa, 2006) and immune response (Klein and Flanagan, 2016; Osborne et al., 2018), the primary goal of the present study was to investigate whether PTSD is associated with sex-specific differences in leukocyte composition, detectable by DNAm-based estimates. We found that males with lifetime PTSD showed significantly higher monocyte proportions than trauma-exposed males without PTSD; this difference was not observed in females. No difference in monocyte proportions was observed between current and remitted PTSD cases in males, suggesting that this sex-specific difference may reflect a long-standing trait of lifetime history of PTSD diagnosis, rather than current state of PTSD. These findings were observed in both the primary RPC and comparison CP-based sets of cell estimates, which were derived using non-constrained vs. constrained projection deconvolution algorithms, respectively. Overall, our main finding of elevated monocyte proportions in males, but not females with lifetime history of PTSD provides evidence for a sex-specific difference in peripheral blood leukocyte composition that may reflect long-standing changes associated with PTSD diagnosis and is detectable in methylomic profiles, consistently across different deconvolution algorithms. 


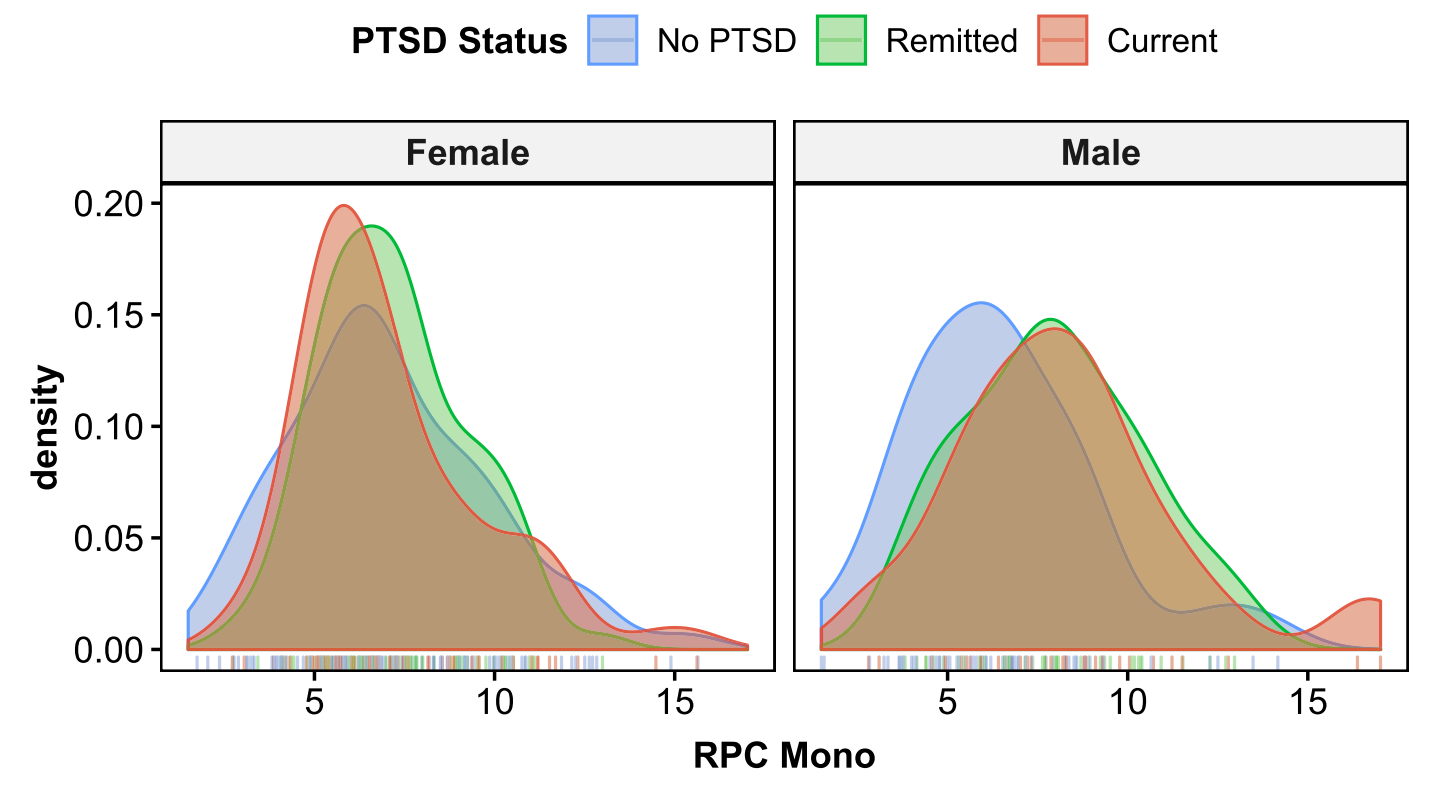

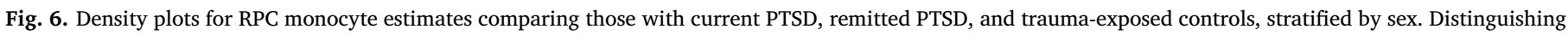

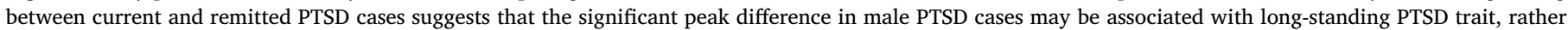

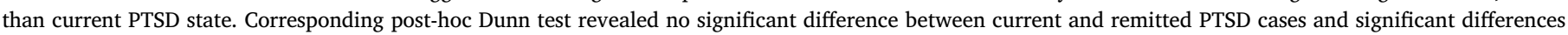
between PTSD case groups and trauma-exposed controls. Again, no significant differences were observed in females.

In our study, we leveraged recent advances in reference-based deconvolution methods (Teschendorff et al., 2017; Koestler, 2016; Salas, 2018) - specifically the EpiDISH algorithm (Teschendorff et al., 2017), which (i) employs DNase hypersensitive site (DHS) data of leukocyte subtypes to inform probe selection for their reference database and (ii) introduces RPC, a non-constrained projection approach for referencebased deconvolution. A comparative validation study on in-silico mixtures of purified cell DNAm profiles previously showed this newer RPC approach to consistently perform better than the widely used CP approach (Houseman, 2012), based on root mean square error (RMSE) and R2, at low noise levels (Teschendorff et al., 2017) typically encountered in real data (Teschendorff et al., 2017; Salas, 2018). Relevant to our results, the study showed the difference in RMSE and R2 to be the most prominent in CD8T cells (Teschendorff et al., 2017). This is consistent with our comparison between RPC and CP estimates, which showed CD8T cells to exhibit poorer correlation between RPC and CP estimates relative to other leukocyte subtypes and the largest difference in RPC-CP correlation between sexes. Similarly, the validation study reported better performance of RPC, compared to $\mathrm{CP}$, in monocytes, with higher RMSE and lower R2 in CP compared to RPC, suggesting RPC-based estimates were more robustly associated with true weights. In light of the validation study, this suggests that our RPC-based estimates were better able to resolve male-specific association of monocyte proportions with lifetime PTSD. In all, our results were consistent with the previously published validation study (Teschendorff et al., 2017) and favored use of RPC estimates for modeling leukocyte composition. However, as these methods have been developed recently, further validation and comparative studies are warranted.

Comparison of leukocyte subtype estimates by sex revealed significant baseline sex differences in the distributions of NK cell and CD8T cell proportions, with males showing greater median NK and lower median CD8T cell proportions, using both RPC and CP based estimates. This finding is consistent with a previous study that reported sex differences in both leukocyte subtypes using estimates based on minfi's implementation of the Houseman approach (Inoshita, 2015) and with immunology literature that reported higher NK cell counts and proportions in males compared to females (Abdullah, 2012). A recent study that modeled cell-specific methylation profiles also reported robust sex differences in $\mathrm{CD}^{+} 6^{+}$NK methylation patterns (White, 2017), suggesting that this leukocyte subtype may be regulated by DNAm in a sex-specific manner. Additionally, DNAm dynamics have been found to drive effector functions in CD8T cells after stimulation (Scharer et al., 2013; Suarez-Alvarez et al., 2012). Development of reference databases that resolve the six main leukocyte subtypes to consider proportions of subsets with shared lineage but different functionality/phenotype (e.g., naïve vs. memory vs. regulatory subtypes) may allow us to explore this hypothesis and would greatly enrich our understanding of immune activity.

Our main finding of higher monocyte proportions in male lifetime PTSD cases is consistent with a previous study of Gulf War Illness (GWI) in a predominantly male veteran cohort, which reported an association between GWI and increase in monocyte count (Johnson et al., 2016). However, comparable studies reporting monocyte counts from differential leukocyte count are generally lacking; the majority of immune studies of PTSD have focused on inflammatory markers (e.g., cytokine levels) and cellular activity, including spontaneous and stimulated cytokine production, and studies of cell counts/proportions have focused on lymphocytes, particularly T-cell subsets (Passos, 2015; Wang and Young, 2016; Speer et al., 2018; Pace and Heim, 2011; Ironson et al., 2007; Wong, 2002). Two PTSD studies that reported monocyte counts based on white blood cell differential count found no significant difference in monocyte proportions (Rohleder et al., 2004; Gola, 2013), which is consistent with our results in both sexes, but did not conduct sex-stratified analyses. Additionally, a small study in adult females that matched PTSD participants and controls for phase of menstrual cycle agreed with our female-specific results and reported no difference between PTSD subjects and controls in percentage of any lymphocyte subsets or total numbers of leukocytes, neutrophils, lymphocytes, or monocytes (Altemus et al., 2006). PTSD studies investigating peripheral lymphocyte numbers have reported mixed findings (Kawamura et al., 2001; Zhou, 2014; Pace and Heim, 2011; Ironson et al., 2007; Rohleder et al., 2004; Boscarino and Chang, 1999; de Kloet, 2007; Vidovic, 2011; Vidovic, 2007; Jergovic, 2014), but more recent studies that further resolved T-cell subpopulations support PTSD-associated differences in T-cell composition indicative of pro-inflammatory skew (Sommershof, 2009; Zhou, 2014; Jergovic, 2014) and immunological aging (Aiello, 2016). Many of these studies were conducted predominantly in one sex (often in male veteran cohorts) (Zhou, 2014; Boscarino and Chang, 
1999; de Kloet, 2007; Vidovic, 2011, 2007; Jergovic, 2014) and those based on both sexes did not conduct sex-stratified a nalyses (Aiello, 2016; Sommershof, 2009; Rohleder et al., 2004). To our knowledge, no authoritative study of sex differences in complete blood counts in PTSD has yet been published, and studies of sex differences in PTSD have generally been lacking, with a number of large-scale studies conducted in predominantly male military cohorts (or on female-only cohorts, e.g. Nurses' Health Study II) (Koenen, 2009; Sumner, 2017).

While not for PTSD, a study of depression that examined white blood cell differential count noted a significant increase in monocyte count and proportions among males with major depressive disorder (MDD), but not females, and a significant s ex by diagnosis i nteraction (Maes, 1992). Likewise, a separate longitudinal study following MDD inpatients also reported elevated monocyte counts in patients compared to controls, and this was driven by men (Seidel, 1996). Additionally, a decrease in depression severity was associated with a decrease in monocyte counts (Seidel, 1996), suggesting that monocytes may be related to clinical improvement. Similarly, the presence and severity of atherosclerosis, another condition linked to PTSD via systemic low-grade inflammatory state (Brouwers et al., 2015), are also associated with an increase in monocyte count in males, but not females (Huang, 2001).

Further prospective investigation of PTSD is needed to determine whether the higher monocyte proportion observed in males reflects an increased susceptibility for developing PTSD or if it reflects a n immunological shift in response to the precipitating trauma associated with PTSD psychopathology. However, studies in a male rodent model provide strong evidence for the latter and have been important for establishing the relationship between peripheral immune cells and the brain in the context of psychosocial stress and associated behavior. Repeated social defeat (RSD) was found to induce myelopoiesis and release inflammatory ( Ly6C $\mathrm{C}^{\mathrm{hi}}$ ) $\mathrm{m}$ onocytes i nto c irculation $\mathrm{v}$ ia s ympathetic signaling, and this increased level of circulating peripheral monocytes was associated with recruitment of pro-inflammatory monocytes/macrophages to the brain and neuroinflammation (Wohleb, 2011; Engler et al., 2004; Weber et al., 2017). Increased proportion of these peripheral monocytes and macrophage recruitment to the brain were also demonstrated to correspond with development, maintenance, and re-establishment of RSD-induced anxiety-like behavior; blockade of this recruitment (via splenectomy or $\beta$-adrenergic receptor blockage) before RSD was found to prevent development of anxiety-like behavior (Wohleb, 2014; McKim, 2016). Additionally, a recent paper discerned that stress-induced anxiety-like behavior and social avoidance are dependent on an increase in IL-6 after stress exposure, which induces a primed transcriptional profile in monocytes recruited to the brain and propagates IL-1 $\beta$ mediated inflammation associated with anxiety-like behavior (Niraula et al., 2019). These studies implicate peripheral monocytes in directly affecting relevant PTSD-like behavior after stress exposure in males (Bierhaus, 2003; Grisanti, 2010). Very recently, the first study using a modified version of the RSD paradigm was conducted in female mice and reported a similar onset of anxiety-like and social avoidance behavior, increase in myelopoiesis, increase in peripheral monocyte proportions, and recruitment of peripheral myeloid cells to the brain, $14 \mathrm{~h}$ after the last RSD cycle (Yin, 2019). Continued work based on this paradigm at multiple time points may be fruitful for investigating if there are sex differences in the kinetics of leukocyte trafficking and ti ssue distribution, especially since recent investigations in other PTSD-relevant rodent models suggest fundamental sex differences in neurobiological response to trauma exposure (Pooley, 2018) and in regulation of stress/trauma-induced neuroinflammatory p riming/neuroimmune a lterations ( Fonken, 2018; Bekhbat and Neigh, 2018). Furthermore, a social stress paradigm in pregnant rats reported lower numbers of monocytes in stressed females than control female rats (Stefanski et al., 2005), illustrating the importance of considering different paradigms and breeds/species.

Although no studies of PTSD have investigated sex differences in monocyte counts or methylomic profiles, chronic PTSD-associated sex differences were noted in transcriptional regulation (O'Donovan, 2011) and gene expression (Neylan, 2011) of $\mathrm{CD}_{14} 4^{+}$monocytes isolated from peripheral blood. Given the inherent sex differences in innate immune response (Klein and Flanagan, 2016; Nunn et al., 2009), as understood in the context of infection, injury, and treatment of inflammatory disorders, sexually dimorphic dynamics and effects may also exist in the context of neuroimmune response to stress/trauma exposure (Bekhbat and Neigh, 2018). One relevant sex difference in monocytes involves the expression of IL-6, which was suggested to be important for stress-induced anxiety-like behavior and social avoidance in the RSD model (Niraula et al., 2019). Independent of reproductive hormones (i.e., estradiol, dehydroepiandrosterone [DHEA], progesterone), women were shown to have greater monocyte expression of IL-6 across a circadian period than men, and sympathovagal balance was negatively associated with monocyte IL- 6 expression only in women (O'Connor et al., 2007). On the other hand, a study examining sex differences in regulation of inflammatory cell recruitment and cytokine synthesis found that ovarian hormones regulate phenotype, function, and numbers of macrophages, but not T lymphocytes, in females (Scotland et al., 2011). This fundamental sex difference may underlie more efficient recognition and elimination of infectious stimuli without recruitment of circulating neutrophils or excessive cytokine production in females, compared to males (Scotland et al., 2011), and may also have implications in the context of psychosocial stress exposure. Relatedly, statins, which have anti-inflammatory activity, modulate monocyte migration in a sex-specific manner, such that both spontaneous and lipopolysaccharide-induced migration of isolated monocytes were found to be inhibited by statins in women, but not men (Ruggieri, 2014).

Our observation of male-specific increase in monocyte proportions associated with lifetime PTSD may reflect fundamental sex differences in leukocyte trafficking, tissue distribution, and thus composition in blood, with implications for stress/trauma-induced neuroimmune alterations and behavior. Of note, while the effect size detected in males translates to an absolute difference of only $1.3 \%$ in monocyte proportions between participants with vs. without PTSD ( $\sim 8.1 \%$ vs. $6.8 \%$; Table 3 ), it also corresponds to an increase of approximately $19 \%$ in overall monocytes among men with lifetime PTSD. Furthermore, the lack of difference between remitted and current PTSD observed in males may have a number of implications for PTSD pathophysiology, including adverse health consequences associated with PTSD across the life course in men, which may be distinct from PTSD-associated health trajectories in women (Dedert et al., 2010; Mitchell, 2013; McLean et al., 2011).

Although the current dataset combined two cohorts and known pregnancies were excluded from our study, sample size and unavailable phenotype data on pregnancy, timing of the menstrual cycle, hormonal birth control use, well-harmonized measures of depression, and health status, as well as gender-related variables, such as coping mechanisms, are all limitations of this study. Future studies that account for hormone levels and other fundamental physiological sex differences may help identify female-specific associations between PTSD and leukocyte composition and clarify if hormone-dependent processes influence leukocyte composition dynamics. Additionally, both cohorts included in this study are civilian, urban, and predominantly African-American, so generalizability of our findings may be limited to this population.

Overall, our study implements current state-of-the-art methods to illustrate feasibility of using DNAm-based leukocyte composition estimates to probe immune profiles. Our literature-supported finding of higher DNAmbased monocyte proportions in males may be an informative metric to include as part of a diagnostic biomarker panel for PTSD in males, and future study in females, with consideration for hormonal status, may elucidate a female-specific panel as well. Differential methylation markers discovered in sex-stratified EWAS, which account for these cell estimates as covariates, are other prime candidates to be included in such sex-specific biomarker panels. Furthermore, in addition to being able to infer leukocyte composition when complete blood count data is not available, these DNAm-based estimates of leukocyte composition can be used to determine cell-specific differential methylation profiles. In fact, methods and validation for cellspecific differential methylation analyses have been published very recently 
(Zheng et al., 2018; Li et al., 2019) and may enable the next significant advance in extracting insights from methylomic profiles by contextualizing how differential methylation in specific leukocyte subtypes alter regulatory dynamics in the immune system. Ultimately, this work may help to shape future studies designed to determine whether sex-specific methylomic metrics of peripheral immune status can inform us about sex differences in neuroinflammation and corresponding behavior in response to trauma exposure.

\section{Conclusion}

By combining DNA methylation datasets from two civilian cohorts, the current study found significantly higher monocyte proportions in males with lifetime PTSD compared to trauma-exposed controls, a difference that was not observed in females. This sex-specific difference in peripheral blood leukocyte composition may reflect a long-standing trait of PTSD diagnosis, rather than current state of PTSD. While this finding was confirmed using two different cell estimation approaches (i.e., deconvolution algorithms), the recently developed non-constrained projection approach (RPC) appears better suited for modeling leukocyte composition. Methylome-based characterization of immune profiles holds special promise for the study of PTSD and continued development of reference databases and validation of methods will build on these recent improvements to enrich our understanding of sexspecific immune dysregulation associated with PTSD.

\section{Funding}

NIH grants: R01MD011728; 3R01MD011728-02S1; R01 DA022720; DA022720-S1; RC1MH088283; MH096764; MH071537; University of Illinois: CompGen Fellowship.

\section{Declaration of Competing Interest}

None.

\section{Acknowledgments}

We appreciate the time and effort of study participants, staff and volunteers of the Detroit Neighborhood Health Study and Grady Trauma Project.

\section{Appendix A. Supplementary data}

Supplementary data associated with this article can be found, in the online version, at http://dx.doi.org/10.1016/j.bbi.2019.06.025.

\section{References}

Abdullah, M., et al., 2012. Gender effect on in vitro lymphocyte subset levels of healthy individuals. Cell Immunol 272, 214-219. https://doi.org/10.1016/j.cellimm.2011. 10.009.

Aiello, A.E., et al., 2016. PTSD is associated with an increase in aged T cell phenotypes in adults living in Detroit. Psychoneuroendocrinology 67, 133-141. https://doi.org/10. 1016/j.psyneuen.2016.01.024.

Altemus, M., Cloitre, M., Dhabhar, F.S., 2003. Enhanced cellular immune response in women with PTSD related to childhood abuse. Am. J. Psychiat. 160, 1705-1707. https://doi.org/10.1176/appi.ajp.160.9.1705.

Altemus, M., Dhabhar, F.S., Yang, R., 2006. Immune function in PTSD. Ann. N Y Acad. Sci. 1071, 167-183. https://doi.org/10.1196/annals.1364.013.

Alvarez-Errico, D., Vento-Tormo, R., Sieweke, M., Ballestar, E., 2015. Epigenetic control of myeloid cell differentiation, identity and function. Nat. Rev. Immunol. 15, 7-17. https://doi.org/10.1038/nri3777.

Aryee, M.J., et al., 2014. Minfi: a flexible and comprehensive Bioconductor package for the analysis of Infinium DNA methylation microarrays. Bioinformatics 30 , 1363-1369. https://doi.org/10.1093/bioinformatics/btu049.

American Psychiatric Association, 1994. Diagnostic and statistical manual of mental disorders. Fourth ed. (American Psychiatric Association, Washington, DC).

American Psychiatric Association, 2013. Diagnostic and statistical manual of mental disorders. 5 ed.

Bam, M., et al., 2016. Dysregulated immune system networks in war veterans with PTSD is an outcome of altered miRNA expression and DNA methylation. Sci. Rep. 6, 31209. https://doi.org/10.1038/srep31209.

Bam, M., et al., 2016. Evidence for epigenetic regulation of pro-inflammatory cytokines, interleukin-12 and interferon gamma, in peripheral blood mononuclear cells from PTSD patients. J. Neuroimmune. Pharmacol. 11, 168-181. https://doi.org/10.1007/ s11481-015-9643-8.

Bangasser, D.A., Valentino, R.J., 2014. Sex differences in stress-related psychiatric disorders: neurobiological perspectives. Front. Neuroendocrinol. 35, 303-319. https:// doi.org/10.1016/j.yfrne.2014.03.008.

Barfield, R.T., et al., 2014. Accounting for population stratification in DNA methylation studies. Genet. Epidemiol. 38, 231-241. https://doi.org/10.1002/gepi.21789.

Barfield, R.T., Kilaru, V., Smith, A.K., Conneely, K.N., 2012. CpGassoc: an R function for analysis of DNA methylation microarray data. Bioinformatics 28, 1280-1281. https://doi.org/10.1093/bioinformatics/bts124.

Bekhbat, M., Neigh, G.N., 2018. Sex differences in the neuro-immune consequences of stress: Focus on depression and anxiety. Brain Behav. Immun. 67, 1-12. https://doi. org/10.1016/j.bbi.2017.02.006.

Benjet, C., et al., 2016. The epidemiology of traumatic event exposure worldwide: results from the World Mental Health Survey Consortium. Psychol. Med. 46, 327-343. https://doi.org/10.1017/S0033291715001981.

Bersani, F.S., et al., 2016. A population of atypical CD56(-)CD16(+) natural killer cells is expanded in PTSD and is associated with symptom severity. Brain Behav. Immun. 56, 264-270. https://doi.org/10.1016/j.bbi.2016.03.021.

Bierhaus, A., et al., 2003. A mechanism converting psychosocial stress into mononuclear cell activation. Proc. Natl. Acad. Sci. U.S.A. 100, 1920-1925. https://doi.org/10. 1073/pnas.0438019100.

Binder, E.B., et al., 2008. Association of FKBP5 polymorphisms and childhood abuse with risk of posttraumatic stress disorder symptoms in adults. JAMA 299, 1291-1305. https://doi.org/10.1001/jama.299.11.1291.

Blake, D.D., et al., 1995. The development of a Clinician-Administered PTSD Scale. J. Trauma Stress 8, 75-90.

Blanchard, E.B., Jones-Alexander, J., Buckley, T.C., Forneris, C.A., 1996. Psychometric properties of the PTSD Checklist (PCL). Behav. Res. Ther. 34, 669-673.

Boscarino, J.A., Chang, J., 1999. Higher abnormal leukocyte and lymphocyte counts 20 years after exposure to severe stress: research and clinical implications. Psychosom. Med. 61, 378-386.

Breslau, N., et al., 1998. Trauma and posttraumatic stress disorder in the community: the 1996 Detroit Area Survey of Trauma. Arch. Gen. Psychiat. 55, 626-632.

Breslau, N., 2009. The epidemiology of trauma, PTSD, and other posttrauma disorders. Trauma Viol. Abuse 10, 198-210. https://doi.org/10.1177/1524838009334448.

Breslau, N., Davis, G.C., Andreski, P., Peterson, E.L., Schultz, L.R., 1997. Sex differences in posttraumatic stress disorder. Arch. Gen. Psychiat. 54, 1044-1048.

Brouwers, C. J., Wolf, J. M. von Känel, R. 2015. Comprehensive Guide to Post-Traumatic Stress Disorder Ch. Chapter 54-1, 1-13.

Chen, Y.A., et al., 2013. Discovery of cross-reactive probes and polymorphic CpGs in the Illumina Infinium HumanMethylation450 microarray. Epigenetics 8, 203-209. https://doi.org/10.4161/epi.23470.

Chen, L., et al., 2016. Genetic drivers of epigenetic and transcriptional variation in human immune cells. Cell 167, 1398-1414. https://doi.org/10.1016/j.cell.2016.10.026.

de Kloet, C.S., et al., 2007. Leukocyte glucocorticoid receptor expression and immunoregulation in veterans with and without post-traumatic stress disorder. Mol. Psychiat. 12, 443-453. https://doi.org/10.1038/sj.mp.4001934.

Dedert, E.A., Calhoun, P.S., Watkins, L.L., Sherwood, A., Beckham, J.C., 2010. Posttraumatic stress disorder, cardiovascular, and metabolic disease: a review of the evidence. Ann. Behav. Med. 39, 61-78. https://doi.org/10.1007/s12160-0109165-9.

Engler, H., Bailey, M.T., Engler, A., Sheridan, J.F., 2004. Effects of repeated social stress on leukocyte distribution in bone marrow, peripheral blood and spleen. J. Neuroimmunol. 148, 106-115. https://doi.org/10.1016/j.jneuroim.2003.11.011.

Fonken, L.K., et al., 2018. Neuroinflammatory priming to stress is differentially regulated in male and female rats. Brain Behav. Immun. 70, 257-267. https://doi.org/10.1016/ j.bbi.2018.03.005.

Fortin, J.P., Triche Jr., T.J., Hansen, K.D., 2017. Preprocessing, normalization and integration of the Illumina HumanMethylationEPIC array with minfi. Bioinformatic 33, 558-560. https://doi.org/10.1093/bioinformatics/btw691.

Gentleman, R.C., et al., 2004. Bioconductor: open software development for computational biology and bioinformatics. Genome Biol. 5, R80. https://doi.org/10.1186/gb2004-5-10-r80.

Gill, J.M., Saligan, L., Woods, S., Page, G., 2009. PTSD is associated with an excess of inflammatory immune activities. Perspect. Psychiat. Care 45, 262-277. https://doi. org/10.1111/j.1744-6163.2009.00229.x.

Gillespie, C.F., et al., 2009. Trauma exposure and stress-related disorders in inner city primary care patients. Gen. Hosp. Psychiat. 31, 505-514. https://doi.org/10.1016/j genhosppsych.2009.05.003.

Glover, D.A., Steele, A.C., Stuber, M.L., Fahey, J.L., 2005. Preliminary evidence for lymphocyte distribution differences at rest and after acute psychological stress in PTSD-symptomatic women. Brain Behav. Immun. 19, 243-251. https://doi.org/10. 1016/j.bbi.2004.08.002.

Gola, H., et al., 2013. Posttraumatic stress disorder is associated with an enhanced spontaneous production of pro-inflammatory cytokines by peripheral blood mononuclear cells. BMC Psychiat. 13. https://doi.org/10.1186/1471-244x-13-40.

Goldmann, E., et al., 2011. Pervasive exposure to violence and posttraumatic stress disorder in a predominantly African American Urban Community: the Detroit Neighborhood Health Study. J. Trauma Stress 24, 747-751. https://doi.org/10. $1002 /$ jts. 20705.

Gotovac, K., et al., 2010. Natural killer cell cytotoxicity and lymphocyte perforin 
expression in veterans with posttraumatic stress disorder. Prog. Neuropsychopharmacol. Biol. Psychiat. 34, 597-604. https://doi.org/10.1016/j. pnpbp.2010.02.018.

Grisanti, L.A., et al., 2010. Pro-inflammatory responses in human monocytes are beta1 adrenergic receptor subtype dependent. Mol. Immunol. 47, 1244-1254. https://doi. org/10.1016/j.molimm.2009.12.013.

Grubaugh, A.L., Elhai, J.D., Cusack, K.J., Wells, C., Frueh, B.C., 2007. Screening for PTSD in public-sector mental health settings: the diagnostic utility of the PTSD checklist. Depress Anxiet. 24, 124-129. https://doi.org/10.1002/da.20226.

Hodes, G.E., 2013. Sex, stress, and epigenetics: regulation of behavior in animal models of mood disorders. Biol. Sex Differ. 4, 1. https://doi.org/10.1186/2042-6410-4-1.

Hoge, E.A., et al., 2009. Broad spectrum of cytokine abnormalities in panic disorder and posttraumatic stress disorder. Depress Anxiety 26, 447-455. https://doi.org/10. 1002/da.20564.

Holm, S., 1979. A simple sequentially rejective multiple test procedure. Scand. J. Statist. 6, 65-70.

Houseman, E.A., et al., 2012. DNA methylation arrays as surrogate measures of cell mixture distribution. BMC Bioinformat. 13, 1-16. https://doi.org/10.1186/1471 2105-13-86.

Huang, Z.S., et al., 2001. Correlations between peripheral differential leukocyte counts and carotid atherosclerosis in non-smokers. Atherosclerosis 158, 431-436. https:// doi.org/10.1016/S0021-9150(01)00445-2.

Huber, W., et al., 2015. Orchestrating high-throughput genomic analysis with bioconductor. Nat. Methods 12, 115-121. https://doi.org/10.1038/nmeth.3252.

Inoshita, M., et al., 2015. Sex differences of leukocytes DNA methylation adjusted for estimated cellular proportions. Biol. Sex Differ. 6, 11. https://doi.org/10.1186/ s13293-015-0029-7.

Ironson, G., Cruess, D., Kumar, M., 2007. in Psychoneuroimmunology (Fourth Edition) (ed Robert Ader) 531-547. Academic Press.

Irwin, M.R., Cole, S.W., 2011. Reciprocal regulation of the neural and innate immune systems. Nat. Rev. Immunol. 11, 625-632. https://doi.org/10.1038/nri3042.

Jergovic, M., et al., 2014. Patients with posttraumatic stress disorder exhibit an altered phenotype of regulatory T cells. Allergy Asthma Clin. Immunol. 10, 43. https://doi. org/10.1186/1710-1492-10-43.

Johnson, W.E., Li, C., Rabinovic, A., 2007. Adjusting batch effects in microarray expression data using empirical Bayes methods. Biostatistics 8, 118-127. https://doi. org/10.1093/biostatistics/kxj037.

Johnson, G.J., Slater, B.C., Leis, L.A., Rector, T.S., Bach, R.R., 2016. Blood biomarkers of chronic inflammation in Gulf War Illness. e0157855. PLoS One 11. https://doi.org/ 10.1371/journal.pone.0157855.

Jovanovic, T., et al., 2010. Impaired fear inhibition is a biomarker of PTSD but not depression. Depress Anxiety 27, 244-251. https://doi.org/10.1002/da.20663.

Kawamura, N., Kim, Y., Asukai, N., 2001. Suppression of cellular immunity in men with a past history of posttraumatic stress disorder. Am. J. Psychiat. 158, 484-486. https:// doi.org/10.1176/appi.ajp.158.3.484

Kessler, R.C., et al., 1994. Lifetime and 12-month prevalence of DSM-III-R psychiatric disorders in the United States. Results from the National Comorbidity Survey. Arch. Gen. Psychiat. 51, 8-19.

Kessler, R.C., et al., 2005. Lifetime prevalence and age-of-onset distributions of DSM-IV disorders in the National Comorbidity Survey Replication. Arch. Gen. Psychiat. 62, 593-602. https://doi.org/10.1001/archpsyc.62.6.593.

Kessler, R.C., McGonagle, K.A., Swartz, M., Blazer, D.G., Nelson, C.B., 1993. Sex and depression in the National Comorbidity Survey. I: Lifetime prevalence, chronicity and recurrence. J. Affect. Disord. 29, 85-96.

Kessler, R.C., Sonnega, A., Bromet, E., Hughes, M., Nelson, C.B., 1995. Posttraumatic stress disorder in the National Comorbidity Survey. Arch Gen Psychiat. 52, 1048-1060.

Kessler, R.C., Wang, P.S., 2008. The descriptive epidemiology of commonly occurring mental disorders in the United States. Annu. Rev. Public Health 29, 115-129. https:// doi.org/10.1146/annurev.publhealth.29.020907.090847.

Kilpatrick, D.G., et al., 2013. National estimates of exposure to traumatic events and PTSD prevalence using DSM-IV and DSM-5 criteria. J. Trauma Stress 26, 537-547. https:// doi.org/10.1002/jts.21848.

Klein, S.L., Flanagan, K.L., 2016. Sex differences in immune responses. Nat. Rev. Immunol. 16, 626-638. https://doi.org/10.1038/nri.2016.90.

Koenen, K.C., et al., 2009. Protocol for investigating genetic determinants of posttraumatic stress disorder in women from the Nurses' Health Study II. BMC Psychiat. 9, 29. https://doi.org/10.1186/1471-244X-9-29.

Koenen, K.C., et al., 2011. SLC6A4 methylation modifies the effect of the number of traumatic events on risk for posttraumatic stress disorder. Depress Anx. 28, 639-647. https://doi.org/10.1002/da.20825.

Koestler, D.C., et al., 2016. Improving cell mixture deconvolution by identifying optimal DNA methylation libraries (IDOL). BMC Bioinformat. 17, 120. https://doi.org/10. 1186/s12859-016-0943-7.

Koestler, D.C., et al., 2017. DNA methylation-derived neutrophil-to-lymphocyte ratio: an epigenetic tool to explore cancer inflammation and outcomes. Cancer Epidemiol. Biomarkers Prev. 26, 328-338. https://doi.org/10.1158/1055-9965.EPI-16-0461.

Kwapis, J.L., Wood, M.A., 2014. Epigenetic mechanisms in fear conditioning: implications for treating post-traumatic stress disorder. Trend. Neurosci. 37, 706-720. https://doi. org/10.1016/j.tins.2014.08.005.

Leek, J.T., Johnson, W.E., Parker, H.S., Jaffe, A.E., Storey, J.D., 2012. The sva package for removing batch effects and other unwanted variation in high-throughput experiments. Bioinformatics 28, 882-883. https://doi.org/10.1093/bioinformatics/bts034.

Li, Z., Wu, Z., Jin, P., Wu, H., 2019. Dissecting differential signals in high-throughput data from complex tissues. Bioinformatics. https://doi.org/10.1093/bioinformatics/ btz196.
Lindqvist, D., et al., 2014. Proinflammatory milieu in combat-related PTSD is independent of depression and early life stress. Brain Behav. Immun. 42, 81-88. https://doi.org/10.1016/j.bbi.2014.06.003.

Lindqvist, D., et al., 2017. Increased pro-inflammatory milieu in combat related PTSD - a new cohort replication study. Brain Behav. Immun. 59, 260-264. https://doi.org/10. 1016/j.bbi.2016.09.012.

Liu, H., et al., 2017. Association of DSM-IV posttraumatic stress disorder with traumatic experience type and history in the world health organization world mental health surveys. JAMA Psychiat. 74, 270-281. https://doi.org/10.1001/jamapsychiatry. 2016.3783.

Liu, J., Siegmund, K.D., 2016. An evaluation of processing methods for HumanMethylation450 BeadChip data. BMC Genom. 17, 469. https://doi.org/10. 1186/s12864-016-2819-7.

Luppi, P., 2003. How immune mechanisms are affected by pregnancy. Vaccine 21, 3352-3357. https://doi.org/10.1016/S0264-410x(03)00331-1.

Maddox, S.A., Schafe, G.E., Ressler, K.J., 2013. Exploring epigenetic regulation of fear memory and biomarkers associated with post-traumatic stress disorder. Front. Psychiat. 4, 62. https://doi.org/10.3389/fpsyt.2013.00062.

Maes, M., et al., 1992. Leukocytosis, monocytosis and neutrophilia: hallmarks of severe depression. J. Psychiatr. Res. 26, 125-134. https://doi.org/10.1016/0022-3956(92) 90004-8.

Malan-Muller, S., Seedat, S., Hemmings, S.M., 2014. Understanding posttraumatic stress disorder: insights from the methylome. Genes Brain Behav. 13, 52-68. https://doi. org/10.1111/gbb.12102.

McGowan, P.O., et al., 2009. Epigenetic regulation of the glucocorticoid receptor in human brain associates with childhood abuse. Nat. Neurosci. 12, 342-348. https:// doi.org/10.1038/nn.2270.

McKim, D.B., et al., 2016a. Sympathetic release of splenic monocytes promotes recurring anxiety following repeated social defeat. Biol. Psychiat. 79, 803-813. https://doi. org/10.1016/j.biopsych.2015.07.010.

McKim, D.B., et al., 2016b. Neuroinflammatory dynamics underlie memory impairments after repeated social defeat. J. Neurosci. 36, 2590-2604. https://doi.org/10.1523/ JNEUROSCI.2394-15.2016.

McLean, C.P., Asnaani, A., Litz, B.T., Hofmann, S.G., 2011. Gender differences in anxiety disorders: prevalence, course of illness, comorbidity and burden of illness. J. Psychiatr. Res. 45, 1027-1035. https://doi.org/10.1016/j.jpsychires.2011.03.006.

Mehta, D., et al., 2013. Childhood maltreatment is associated with distinct genomic and epigenetic profiles in posttraumatic stress disorder. Proc. Natl. Acad. Sci. U.S.A. 110, 8302-8307. https://doi.org/10.1073/pnas.1217750110.

Meyers, J.L., et al., 2015. Frequency of alcohol consumption in humans; the role of metabotropic glutamate receptors and downstream signaling pathways. e586. Transl. Psychiat. 5. https://doi.org/10.1038/tp.2015.70.

Michopoulos, V., Norrholm, S.D., Jovanovic, T., 2015. Diagnostic biomarkers for posttraumatic stress disorder: promising horizons from translational neuroscience research. Biol. Psychiat. 78, 344-353. https://doi.org/10.1016/j.biopsych.2015.01. 005 .

Michopoulos, V., Powers, A., Gillespie, C.F., Ressler, K.J., Jovanovic, T., 2017. Inflammation in fear- and anxiety-based disorders: PTSD, GAD, and beyond. Neuropsychopharmacology 42, 254-270. https://doi.org/10.1038/npp.2016.146.

Miller, C.A., Campbell, S.L., Sweatt, J.D., 2008. DNA methylation and histone acetylation work in concert to regulate memory formation and synaptic plasticity. Neurobiol. Learn Mem. 89, 599-603. https://doi.org/10.1016/j.nlm.2007.07.016.

Mitchell, K.S., et al., 2013. PTSD and obesity in the Detroit neighborhood health study. Gen. Hosp. Psychiat. 35, 671-673. https://doi.org/10.1016/j.genhosppsych.2013.07. 015.

Morris, T.J., et al., 2014. ChAMP: 450k chip analysis methylation pipeline. Bioinformatics 30, 428-430. https://doi.org/10.1093/bioinformatics/btt684.

Neylan, T.C., et al., 2011. Suppressed monocyte gene expression profile in men versus women with PTSD. Brain Behav. Immun. 25, 524-531. https://doi.org/10.1016/j. bbi.2010.12.001.

Niraula, A., Witcher, K.G., Sheridan, J.F., Godbout, J.P., 2019. Interleukin-6 Induced by social stress promotes a unique transcriptional signature in the monocytes that facilitate anxiety. Biol. Psychiat. 85, 679-689. https://doi.org/10.1016/j.biopsych. 2018.09.030.

Nunn, C.L., Lindenfors, P., Pursall, E.R., Rolff, J., 2009. On sexual dimorphism in immune function. Philos. Trans. R Soc. Lond. B Biol. Sci. 364, 61-69. https://doi.org/10. 1098/rstb.2008.0148.

O'Connor, M.F., Motivala, S.J., Valladares, E.M., Olmstead, R., Irwin, M.R., 2007. Sex differences in monocyte expression of IL-6: role of autonomic mechanisms. Am. J. Physiol. Regul. Integr. Comp. Physiol. 293, R145-151. https://doi.org/10.1152/ ajpregu.00752.2006.

O'Donovan, A., et al., 2011. Transcriptional control of monocyte gene expression in posttraumatic stress disorder. Dis. Markers 30, 123-132. https://doi.org/10.3233/DMA2011-0768.

Osborne, B.F., Turano, A., Schwarz, J.M., 2018. Sex differences in the neuroimmune system. Curr. Opin. Behav. Sci. 23, 118-123. https://doi.org/10.1016/j.cobeha 2018.05.007.

Pace, T.W., Heim, C.M., 2011. A short review on the psychoneuroimmunology of posttraumatic stress disorder: from risk factors to medical comorbidities. Brain Behav. Immun. 25, 6-13. https://doi.org/10.1016/j.bbi.2010.10.003.

Passos, I.C., et al., 2015. Inflammatory markers in post-traumatic stress disorder: a systematic review, meta-analysis, and meta-regression. Lancet Psychiat. 2, 1002-1012. https://doi.org/10.1016/S2215-0366(15)00309-0.

Pfau, M.L., Russo, S.J., 2015. Peripheral and central mechanisms of stress resilience. Neurobiol. Stress 1, 66-79. https://doi.org/10.1016/j.ynstr.2014.09.004.

Pooley, A.E., et al., 2018. Sex differences in the traumatic stress response: PTSD 
symptoms in women recapitulated in female rats. Biol. Sex Differ. 9, 31. https://doi. org/10.1186/s13293-018-0191-9.

R: A Language and Environment for Statistical Computing (R Foundation for Statistical Computing, Vienna, Austria, 2018)

Rahmani, E., et al., 2017. Genome-wide methylation data mirror ancestry information. Epigenetics Chromatin 10, 1. https://doi.org/10.1186/s13072-016-0108-y.

Ratanatharathorn, A., et al., 2017. Epigenome-wide association of PTSD from heterogeneous cohorts with a common multi-site analysis pipeline. Am. J. Med. Genet. B Neuropsychiatr. Genet. 174, 619-630. https://doi.org/10.1002/ajmg.b.32568.

Roadmap Epigenomics, C., et al., 2015. Integrative analysis of 111 reference human epigenomes. Nature 518, 317-330. https://doi.org/10.1038/nature14248.

Rohleder, N., Joksimovic, L., Wolf, J.M., Kirschbaum, C., 2004. Hypocortisolism and increased glucocorticoid sensitivity of pro-Inflammatory cytokine production in Bosnian war refugees with posttraumatic stress disorder. Biol. Psychiat. 55, 745-751. https://doi.org/10.1016/j.biopsych.2003.11.018.

Ruggieri, A., et al., 2014. Statin-induced impairment of monocyte migration is genderrelated. J. Cell Physiol. 229, 1990-1998. https://doi.org/10.1002/jcp.24657.

Ruggiero, K.J., Del Ben, K., Scotti, J.R., Rabalais, A.E., 2003. Psychometric properties of the PTSD Checklist-civilian version. J. Trauma Stress 16, 495-502. https://doi.org/ 10.1023/A:1025714729117.

Rusiecki, J.A., et al., 2013. PTSD and DNA methylation in select immune function gene promoter regions: a repeated measures case-control study of U.S. military service members. Front. Psychiat. 4, 56. https://doi.org/10.3389/fpsyt.2013.00056.

Salas, L.A., et al., 2018. An optimized library for reference-based deconvolution of wholeblood biospecimens assayed using the Illumina HumanMethylationEPIC BeadArray. Genome Biol. 19, 64. https://doi.org/10.1186/s13059-018-1448-7.

Scharer, C.D., Barwick, B.G., Youngblood, B.A., Ahmed, R., Boss, J.M., 2013. Global DNA methylation remodeling accompanies CD8 T cell effector function. J. Immunol. 191, 3419-3429. https://doi.org/10.4049/jimmunol.1301395.

Scotland, R.S., Stables, M.J., Madalli, S., Watson, P., Gilroy, D.W., 2011. Sex differences in resident immune cell phenotype underlie more efficient acute inflammatory responses in female mice. Blood 118, 5918-5927. https://doi.org/10.1182/blood2011-03-340281.

Seidel, A., et al., 1996. Major depressive disorder is associated with elevated monocyte counts. Acta Psychiat. Scand. 94, 198-204. https://doi.org/10.1111/j.1600-0447. 1996.tb09849.x.

Smith, A.K., et al., 2011. Differential immune system DNA methylation and cytokine regulation in post-traumatic stress disorder. Am. J. Med. Genet. B Neuropsychiat. Genet. 156B, 700-708. https://doi.org/10.1002/ajmg.b.31212.

Sommershof, A., et al., 2009. Substantial reduction of naive and regulatory T cells following traumatic stress. Brain Behav. Immun. 23, 1117-1124. https://doi.org/10. 1016/j.bbi.2009.07.003.

Sondergaard, H.P., Hansson, L.O., Theorell, T., 2004. The inflammatory markers C-reactive protein and serum amyloid $\mathrm{A}$ in refugees with and without posttraumatic stress disorder. Clin. Chim. Acta 342, 93-98. https://doi.org/10.1016/j.cccn.2003.12.019.

Speer, K., Upton, D., Semple, S., McKune, A., 2018. Systemic low-grade inflammation in post-traumatic stress disorder: a systematic review. J. Inflamm. Res. 11, 111-121. https://doi.org/10.2147/JIR.S155903.

Stankiewicz, A.M., Swiergiel, A.H., Lisowski, P., 2013. Epigenetics of stress adaptations in the brain. Brain Res. Bull. 98, 76-92. https://doi.org/10.1016/j.brainresbull.2013. 07.003.

Stefanski, V., Raabe, C., Schulte, M., 2005. Pregnancy and social stress in female rats: influences on blood leukocytes and corticosterone concentrations. J. Neuroimmunol. 162, 81-88. https://doi.org/10.1016/j.jneuroim.2005.01.011.

Suarez-Alvarez, B., Rodriguez, R.M., Fraga, M.F., Lopez-Larrea, C., 2012. DNA methylation: a promising landscape for immune system-related diseases. Trends Genet. 28, 506-514. https://doi.org/10.1016/j.tig.2012.06.005.

Sumner, J.A., et al., 2017. Cross-sectional and longitudinal associations of chronic posttraumatic stress disorder with inflammatory and endothelial function markers in women. Biol. Psychiat. 82, 875-884. https://doi.org/10.1016/j.biopsych.2017.06. 020 .

Sun, B., et al., 2016. DNA methylation perspectives in the pathogenesis of autoimmune diseases. Clin. Immunol. 164, 21-27. https://doi.org/10.1016/j.clim.2016.01.011.

Teschendorff, A.E., et al., 2013. A beta-mixture quantile normalization method for correcting probe design bias in Illumina Infinium $450 \mathrm{k}$ DNA methylation data. Bioinformatics 29, 189-196. https://doi.org/10.1093/bioinformatics/bts680.

Teschendorff, A.E., Breeze, C.E., Zheng, S.C., Beck, S., 2017. A comparison of referencebased algorithms for correcting cell-type heterogeneity in Epigenome-Wide association studies. BMC Bioinformat. 18, 105. https://doi.org/10.1186/s12859-0171511-5.

Tian, Y., et al., 2017. ChAMP: updated methylation analysis pipeline for Illumina BeadChips. Bioinformatics 33, 3982-3984. https://doi.org/10.1093/bioinformatics/ btx513.

Titus, A.J., Gallimore, R.M., Salas, L.A., Christensen, B.C., 2017. Cell-type deconvolution from DNA methylation: a review of recent applications. Hum. Mol. Genet. 26, R216-R224. https://doi.org/10.1093/hmg/ddx275.

Tolin, D.F., Foa, E.B., 2006. Sex differences in trauma and posttraumatic stress disorder: a quantitative review of 25 years of research. Psychol. Bull. 132, 959-992. https://doi. org/10.1037/0033-2909.132.6.959.

Triche Jr., T.J., Weisenberger, D.J., Van Den Berg, D., Laird, P.W., Siegmund, K.D., 2013. Low-level processing of Illumina Infinium DNA Methylation BeadArrays. e90. Nucl.
Acids Res. 41. https://doi.org/10.1093/nar/gkt090.

Uddin, M., et al., 2010. Epigenetic and immune function profiles associated with posttraumatic stress disorder. Proc. Natl. Acad. Sci. U.S.A. 107, 9470-9475. https://doi org/10.1073/pnas.0910794107.

Uddin, M., et al., 2011. Gene expression and methylation signatures of MAN2C1 are associated with PTSD. Dis Mark. 30, 111-121. https://doi.org/10.3233/DMA-20110750 .

Uddin, M., et al., 2018. Epigenetic meta-analysis across three civilian cohorts identifies NRG1 and HGS as blood-based biomarkers for post-traumatic stress disorder. Epigenomics 10, 1585-1601. https://doi.org/10.2217/epi-2018-0049.

Vidovic, A., et al., 2007. Circulating lymphocyte subsets, natural killer cell cytotoxicity, and components of hypothalamic-pituitary-adrenal axis in Croatian war veterans with posttraumatic stress disorder: cross-sectional study. Croat. Med. J. 48, 198-206.

Vidovic, A., et al., 2011. Repeated assessments of endocrine- and immune-related changes in posttraumatic stress disorder. Neuroimmunomodulation 18, 199-211. https://doi. org $/ 10.1159 / 000322869$.

Wang, Z., Young, M.R., 2016. PTSD, a disorder with an immunological component. Front. Immunol. 7, 219. https://doi.org/10.3389/fimmu.2016.00219.

Wang, Z., Mandel, H., Levingston, C.A., Young, M.R.I., 2016. An exploratory approach demonstrating immune skewing and a loss of coordination among cytokines in plasma and saliva of Veterans with combat-related PTSD. Hum. Immunol. 77, 652-657. https://doi.org/10.1016/j.humimm.2016.05.018.

Weathers, F.W., Keane, T.M., Davidson, J.R., 2001. Clinician-administered PTSD scale: a review of the first ten years of research. Depress Anxiety 13, 132-156.

Weaver, I.C., et al., 2004. Epigenetic programming by maternal behavior. Nat. Neurosci. 7, 847-854. https://doi.org/10.1038/nn1276.

Weber, M.D., Godbout, J.P., Sheridan, J.F., 2017. Repeated Social Defeat, Neuroinflammation, and Behavior: Monocytes Carry the Signal. Neuropsychopharmacology 42, 46-61. https://doi.org/10.1038/npp.2016.102.

Weckle, A., et al., 2015. Rapid fractionation and isolation of whole blood components in samples obtained from a community-based setting. J. Vis. Exp. https://doi.org/10. $3791 / 52227$.

White, N., et al., 2017. Accounting for cell lineage and sex effects in the identification of cell-specific DNA methylation using a Bayesian model selection algorithm. e0182455. PLoS One 12. https://doi.org/10.1371/journal.pone.0182455.

Wiencke, J.K., et al., 2017. Immunomethylomic approach to explore the blood neutrophil lymphocyte ratio (NLR) in glioma survival. Clin. Epigenet. 9, 10. https://doi.org/10. 1186/s13148-017-0316-8.

Wilkins, K.C., Lang, A.J., Norman, S.B., 2011. Synthesis of the psychometric properties of the PTSD checklist (PCL) military, civilian, and specific versions. Depress Anxiet. 28, 596-606. https://doi.org/10.1002/da.20837.

Wohleb, E.S., et al., 2011. beta-Adrenergic receptor antagonism prevents anxiety-like behavior and microglial reactivity induced by repeated social defeat. J. Neurosci. 31, 6277-6288. https://doi.org/10.1523/JNEUROSCI.0450-11.2011.

Wohleb, E.S., et al., 2014. Re-establishment of anxiety in stress-sensitized mice is caused by monocyte trafficking from the spleen to the brain. Biol. Psychiat. 75, 970-981. https://doi.org/10.1016/j.biopsych.2013.11.029.

Wohleb, E.S., Delpech, J.C., 2017. Dynamic cross-talk between microglia and peripheral monocytes underlies stress-induced neuroinflammation and behavioral consequences. Prog. Neuropsychopharmacol. Biol. Psychiat. 79, 40-48. https://doi.org/ 10.1016/j.pnpbp.2016.04.013.

Wohleb, E.S., Powell, N.D., Godbout, J.P., Sheridan, J.F., 2013. Stress-induced recruitment of bone marrow-derived monocytes to the brain promotes anxiety-like behavior. J. Neurosci. 33, 13820-13833. https://doi.org/10.1523/JNEUROSCI.1671-13. 2013.

Wolf, E.J., et al., 2018. Traumatic stress and accelerated DNA methylation age: a metaanalysis. Psychoneuroendocrinology 92, 123-134. https://doi.org/10.1016/j. psyneuen.2017.12.007.

Wong, C.M., 2002. Post-traumatic stress disorder: advances in psychoneuroimmunology. Psychiatr. Clin. North Am. 25 369-383,vii.

Xu, Z., Niu, L., Li, L., Taylor, J.A., 2016. ENmix: a novel background correction method for Illumina HumanMethylation450 BeadChip. e20. Nucl. Acids Res. 44. https://doi. org/10.1093/nar/gkv907.

Yehuda, R., Koenen, K.C., Galea, S., Flory, J.D., 2011. The role of genes in defining a molecular biology of PTSD. Dis. Markers 30, 67-76. https://doi.org/10.3233/DMA2011-0794.

Yin, W., et al., 2019. Repeated social defeat in female mice induces anxiety-like behavior associated with enhanced myelopoiesis and increased monocyte accumulation in the brain. Brain. Behav Immun. 78, 131-142. https://doi.org/10.1016/j.bbi.2019.01. 015 .

Zannas, A.S., et al., 2015. Lifetime stress accelerates epigenetic aging in an urban, African American cohort: relevance of glucocorticoid signaling. Genome Biol. 16, 266. https://doi.org/10.1186/s13059-015-0828-5.

Zheng, S.C., Breeze, C.E., Beck, S., Teschendorff, A.E., 2018. Identification of differentially methylated cell types in epigenome-wide association studies. Nat. Methods 15 1059-1066. https://doi.org/10.1038/s41592-018-0213-x.

Zhou, J., et al., 2014. Dysregulation in microRNA expression is associated with alterations in immune functions in combat veterans with post-traumatic stress disorder. e94075. PLoS One 9. https://doi.org/10.1371/journal.pone.0094075.

Zovkic, I.B., Sweatt, J.D., 2013. Epigenetic mechanisms in learned fear: implications for PTSD. Neuropsychopharmacology 38, 77-93. https://doi.org/10.1038/npp.2012.79. 